ARTICLE

\title{
Orthogonal translation enables heterologous ribosome engineering in E. coli
}

\author{
Natalie S. Kolber (10 1,2, Ranan Fattal', Sinisa Bratulic (10 1,3, Gavriela D. Carver (1) ${ }^{1} \&$ Ahmed H. Badran (D) ${ }^{1 凶}$
}

The ribosome represents a promising avenue for synthetic biology, but its complexity and essentiality have hindered significant engineering efforts. Heterologous ribosomes, comprising rRNAs and r-proteins derived from different microorganisms, may offer opportunities for novel translational functions. Such heterologous ribosomes have previously been evaluated in E. coli via complementation of a genomic ribosome deficiency, but this method fails to guide the engineering of refractory ribosomes. Here, we implement orthogonal ribosome binding site (RBS):antiRBS pairs, in which engineered ribosomes are directed to researcherdefined transcripts, to inform requirements for heterologous ribosome functionality. We discover that optimized rRNA processing and supplementation with cognate r-proteins enhances heterologous ribosome function for rRNAs derived from organisms with $\geq 76.1 \%$ 165 rRNA identity to E. coli. Additionally, some heterologous ribosomes undergo reduced subunit exchange with E. coli-derived subunits. Cumulatively, this work provides a general framework for heterologous ribosome engineering in living cells.

\footnotetext{
${ }^{1}$ Broad Institute of MIT and Harvard, Cambridge, MA 02142, USA. ${ }^{2}$ Present address: Department of Bioengineering, Stanford University, Stanford, CA 94305, USA. ${ }^{3}$ Present address: Department of Biology and Biological Engineering, Chalmers University of Technology, Kemivägen 10, SE-412 96 Gothenburg, Sweden.

凶email: ahbadran@broadinstitute.org
} 
$\mathrm{n}$ addition to catalyzing the biosynthesis of the complete cellular proteome, the ribosome serves as a hub for signaling events, integrating nutrient availability with growth dynamics and resource allocation ${ }^{1,2}$. In prokaryotes, this functionality is enabled by the concerted action of numerous components: the $16 \mathrm{~S}$ rRNA and 21 ribosomal proteins (r-proteins) define the small subunit (SSU or $30 \mathrm{~S}$ ), with the $23 \mathrm{~S}$ rRNA, 5S rRNA, and 33 $\mathrm{r}$-proteins defining the large subunit (LSU or $50 \mathrm{~S})^{3}$. Extensive efforts towards engineering translation have yielded researcherdictated, specialized functions in vivo: parallel genetic circuits ${ }^{4}$, augmented polypeptide diversity using non-canonical amino acids $^{5}$, expanded genetic codes incorporating quadruplet codons ${ }^{6}$, and linked ribosomal subunits for improved cellular orthogonality $^{7-9}$. However, these efforts have typically made use of solely E. coli components.

Conversely, ribosomal components derived from divergent microorganisms may offer the opportunity to discover unique or dedicated ribosomal capabilities, as suggested by naturally occurring subpopulations of prokaryotic ribosomes ${ }^{10}$. Indeed, stress-inducible production of $r r s H$ ribosomes in $E$. coli modifies the cellular translational program ${ }^{11}, r r n I$ ribosomes in Vibrio vulnificius selectively translate certain mRNAs ${ }^{12}$, and genetically heterogeneous ribosomes are produced at defined stages of the Streptomyces coelicolor developmental cycle ${ }^{13}$. R-protein complements may further specialize ribosomal function, exemplified by the hypothesized role of ribosomes carrying $\mathrm{S} 1$ in leaderless mRNA decoding ${ }^{14}$ and the variation in r-protein complements during different growth conditions ${ }^{15}$. Heterologous ribosomes, synthesized in E. coli using rRNAs and r-proteins derived from divergent bacterial species, may therefore serve as foundations for the discovery or engineering of novel translational capabilities.

Prior investigations of heterologous rRNAs have been enabled by $E$. coli $\Delta 7$ strains lacking all seven chromosomal rRNA operons (e.g., SQ171, KT101, SQZ10, SQ2518) ${ }^{16-18}$. Such $\Delta 7$ strains have additionally informed studies on rDNA copy number ${ }^{19}$, ribosomal sequence-function relationships ${ }^{20}$, factors affecting rRNA processing ${ }^{21,22}$, and rRNA-protein interactions ${ }^{23}$. These strains bear a complete genomic rRNA deficiency and are complemented by a counter-selectable rRNA-encoding plasmid, facilitating plasmid exchange with rRNA variants capable of sustaining E. coli survival. Promisingly, full-length heterologous rRNA operons derived from species bearing $\geq 93.2 \% 16 \mathrm{~S}$ sequence identity to the $E$. coli counterpart have been found to sustain $E$. coli $\Delta 7$ strain viability ${ }^{18}$, whereas $16 \mathrm{~S}$ sequence fragments bearing $\geq 80.9 \%$ identity can substitute for otherwise wild-type $E$. coli $16 \mathrm{~S}$ rRNAs ${ }^{17}$. Natural horizontal gene transfer events in the evolutionary record provide further evidence for heterologous translation with intragenomic $16 \mathrm{~S}$ identity as low as $88.4 \% \%^{24-28}$.

However, E. coli $\Delta 7$ strain complementation assays prove problematic for systematically evaluating heterologous ribosome function given the myriad roles played by the ribosome in sustaining cell viability, both catalytic and regulatory ${ }^{29,30}$. Efforts to engineer heterologous ribosome function require a more quantitative translational assay that reports on a single aspect of ribosome function. We therefore sought to develop guidelines for evaluating and enhancing the translational activity of heterologous rRNAs in E. coli using a method that reports exclusively on catalytic activity, independent of an rRNA's ability to support cell growth. To achieve this, we first constructed a library of 34 complete rRNA operons derived from phylogenetically diverse microbes. We evaluated their activities via E. coli $\Delta 7$ strain complementation as well as orthogonal translation. Here, engineered RBS:antiRBS pairs are leveraged to generate ribosomes that exclusively translate a researcher-defined transcript, which in turn cannot be translated by wildtype ribosomes ${ }^{4,31-34}$. Finding a high degree of correlation between the two methods, we applied orthogonal translation to guide engineering efforts of rRNA processing sequences and show that divergent intergenic sequences can have significant consequences on heterologous ribosome function in E. coli. Furthermore, we identified a small subset of r-proteins that enhance the activity of refractory heterologous ribosomes with as little as $76.1 \%$ 16S rRNA sequence identity to E. coli. Finally, we find evidence that some heterologous $16 \mathrm{~S}$ rRNAs may preferentially associate with their cognate $23 \mathrm{~S}$ rRNAs in E. coli. Together, these results establish a quantitative and extensible method for the engineering of heterologous ribosome activity in vivo, facilitating the development of diverse ribosomes for synthetic biology applications.

\section{Results}

Heterologous rRNA operons complement SQ171 deficiency. SQ171 is an E. coli strain lacking all seven chromosomal rRNA operons and carrying a single, counter-selectable plasmid bearing the wild-type $r r n C$ operon ${ }^{18,35}$. To investigate the ability of heterologous rRNAs to support SQ171 cell survival, episomally encoded rRNA operons can be introduced into the strain followed by sucrose counterselection of the resident $E$. coli rrnC plasmid using the B. subtilis $s a c B$ cassette (Fig. 1a). Heterologous rRNA operons that yield functional heterologous ribosomes sustain SQ171 growth following sucrose counterselection. Prior work in SQ171 complementation using fully native heterologous rRNA operons has been extended to Salmonella typhimurium (96.8\% 16S rRNA sequence identity to E. coli) and Proteus vulgaris rRNA $(93.2 \%)^{18}$.

We validated this strategy using a number of increasingly divergent heterologous rRNA operons: Salmonella enterica (97.0\% 16S rRNA sequence identity to E. coli), Alteromonas macleodii (85.9\%), Pseudomonas aeruginosa (85.2\%), and Acinetobacter baumannii (84.3\%). Heterologous rRNA derived from $S$. enterica robustly supported SQ171 strain growth, while rRNA derived from $A$. macleodii and $P$. aeruginosa supported growth with a minor defect (Fig. 1b). Surprisingly, we observed a substantial growth defect in SQ171 cells complemented by rRNA derived from $A$. baumannii despite the minor difference in sequence identity to $E$. coli rRNA as compared to $P$. aeruginosa. Motivated by these results, we extended our strategy to a total of 21 increasingly divergent rRNA operons from diverse proteobacterial species. Each of these, including an rRNA derived from the zetaproteobacteria Mariprofundus ferrooxydans (80.7\%), sustained SQ171 growth (Supplementary Table 1). In line with our findings using completely native rRNA operons, chimeric heterologous- $E$. coli $16 \mathrm{~S}$ rRNA fragments from gammaproteobacterial and betaproteobacterial rRNAs can often support $\Delta 7$ strain survival ${ }^{17}$. We further observed a linear relationship between complemented SQ171 strain fitness and 16S rRNA sequence identity, consistent with prior reports that strains relying on increasingly divergent rRNAs show comparatively reduced fitness (Fig. 1c) ${ }^{17,18}$.

Orthogonal translation enables quantitative heterologous ribosome assessment. SQ171 complementation informs the capacity of a heterologous rRNA to translate the E. coli proteome of $>4000$ proteins $^{36}$ as well as the fulfillment of extracatalytic roles, including integrating environmental cues to modulate translation ${ }^{30}$ and initiating the stringent response to cellular stressors ${ }^{29,37}$. Additionally, we observed that SQ171 complementation pipelines require up to 5 days to observe colonies for strains relying on highly divergent rRNAs, where transformed colonies must be laboriously counter-screened due to the high escape frequency of SacB-dependent negative selection (Supplementary Fig. 1) 38,39 . 
a

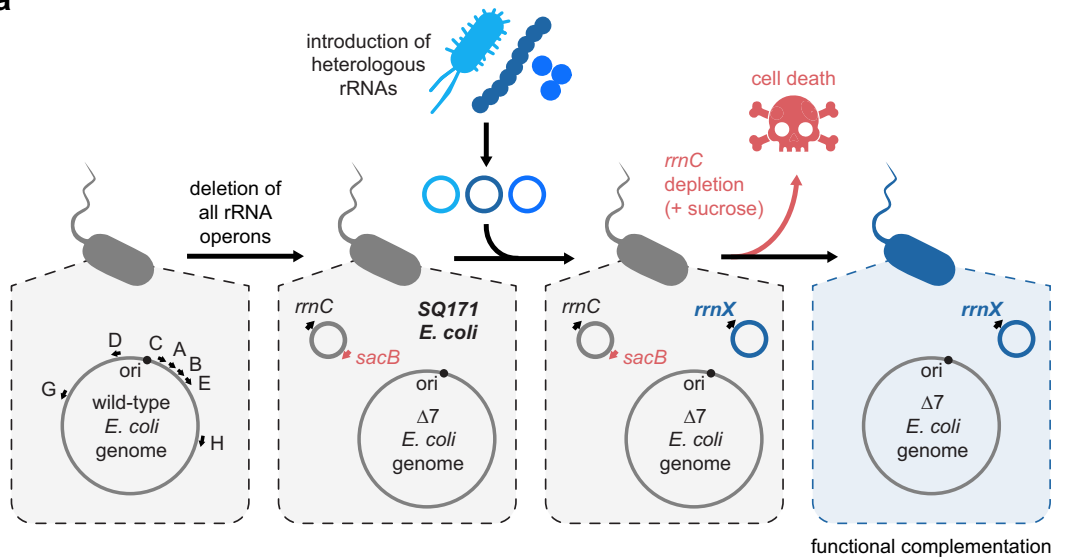

b
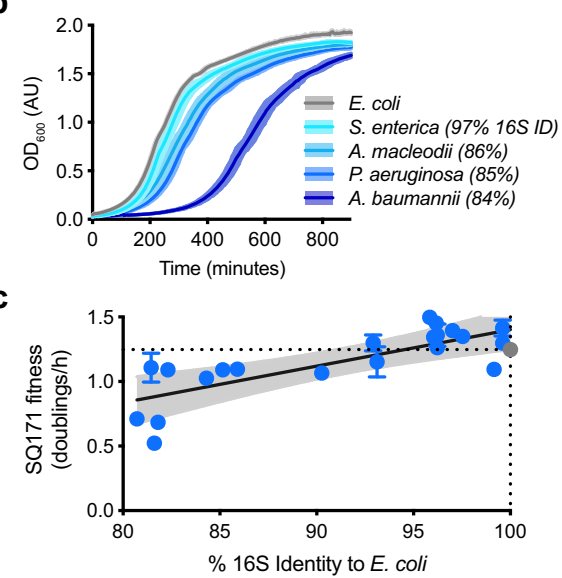

Fig. 1 Assessment of heterologous rRNA activity via SQ171 complementation. a Schematic representation of the SQ171 complementation assay. SQ171 E. coli cells lack all 7 genomic ribosomal RNA (rRNA) operons and maintain a single rrnC operon on a SacB counter-selectable plasmid. Introduction of a heterologous rRNA $(r r n X)$ and depletion of the E. coli rrnC plasmid using sucrose yields cells that rely upon the heterologous ribosome for survival. b Growth time course of SQ171 cells bearing increasingly divergent heterologous rRNAs $(n=2-8)$. c Correlation between heterologous $16 \mathrm{~S}$ rRNA sequence identity to $E$. coli (\%) and SQ171 fitness (doublings/h) upon complementation $\left(99 \% \mathrm{Cl}, R^{2}=0.62\right)$. E. coli rRNA control plotted in gray. Data represent the means of 1-8 biological replicates; error bars represent standard deviations for conditions with 3 or more replicates. Complete SQ171 complementation data (including precise number of replicates) are reported in Supplementary Table 1. OD optical density, AU arbitrary units, h hour. Source data are available in the Source Data File.

To overcome these technical limitations, we sought to develop an assay that delivers a single, quantifiable, translational output that is orthogonal to native ribosomal machinery and therefore independent of cell viability. We leveraged previously described orthogonal ribosome-mRNA pairs, in which the antiRBS of the $16 \mathrm{~S}$ rRNA is engineered to exclusively translate a researcherdefined transcript bearing a complementary $\mathrm{RBS}^{4,8,31-34}$. This yields an orthogonal pool of ribosomes (O-ribosomes) in vivo, whose functions can be easily monitored and quantified via reporter expression (superfolder GFP; $\mathrm{sGFP}^{40}$ ) independent of cellular survival (Fig. 2a). Importantly, wildtype ribosomes are unable to translate the orthogonal mRNA (O-mRNA) reporter, ensuring that the observed reporter activity is dependent upon engineered O-ribosomes (Fig. 2b). This orthogonal translation genetic circuit does not considerably affect cellular viability (Supplementary Fig. 2a) ${ }^{4}$, and both O-mRNA and O-ribosome expression can be controlled via small molecule inducers to further limit the cellular burden of their production (Fig. 2a, c). We extended orthogonal translation to numerous reporter proteins, observing reporter-specific limitations on fluorescent protein functionality (Supplementary Fig. 2b-h). We accordingly identified a ten amino acid sfGFP-derived leader sequence that obviates these constraints and improves orthogonal translation for various reporters (Supplementary Fig. 3a-p).

With a robust reporter system in hand, we engineered the $\mathrm{O}$ antiRBS into all 21 heterologous rRNAs capable of complementing SQ171 viability alongside an additional 13 phylogenetically more divergent rRNAs (Supplementary Table 1). We quantified the activity of all 34 O-rRNAs via orthogonal translation, finding that most rRNAs capable of supporting SQ171 growth similarly synthesized sfGFP at robust levels (Fig. 2d), with the exception of O-rRNAs derived from Serratia marcescens (96.0\% 16S rRNA sequence identity to $E$. coli), Vibrio cholerae (90.3\%), $P$. aeruginosa (85.2\%), A. baumannii (84.3\%), Alcaligenes faecalis (82.3\%), Bordetella pertussis (81.6\%), Burkholderia cenocepacia (81.5\%), and M. ferrooxydans (80.7\%). Notably, sfGFP translation fell markedly with phylogenetic distance from $E$. coli, wherein heterologous rRNAs exclusively derived from gammaproteobacteria and betaproteobacteria were capable of translating sfGFP
(Fig. 2d). Supporting this observation, we found a correlation between 16S rRNA sequence identity to $E$. coli and orthogonal translation activity (Fig. 2e). We similarly observed a correlation between complemented SQ171 fitness and orthogonal translation activity for each functional heterologous rRNA (Fig. 2f). Collectively, these findings support the use of orthogonal translation in lieu of SQ171 complementation to quantify the translational activity of heterologous ribosomes.

Engineered rRNA processing improves heterologous ribosome activity. The observed relationship between heterologous orthogonal translation activity and phylogenetic distance from $E$. coli suggested that certain elements encoded within the rRNA operon may have sufficiently diverged to restrict efficient ribosome assembly in E. coli. Analysis of per-base conservation ${ }^{41}$ across the complete rRNA operons showed appreciably higher conservation scores within the ribosomal genes (16S, 23S, and 5S rRNAs) as compared to intergenic elements (Fig. 3a). Intergenic sequences flanking each rRNA gene are crucial to ribosome biogenesis as they direct pre-rRNA transcript folding and processing by RNases $3,42,43$. We hypothesized that $E$. coli RNases may fail to recognize divergent sequences on non-native rRNA transcripts, yielding immature or poorly processed heterologous ribosomes (Fig. 3b). In this case, substitution of these elements with their $E$. coli counterparts may correct the rRNA processing defect and improve overall orthogonal translation activity.

To assess the impact of putative rRNA processing on heterologous ribosome function in $E$. coli, we substituted the native intergenic sequences of each of the 34 O-rRNAs with their corresponding E. coli sequences (Fig. 3b). Substitution of intergenic sequences for O-rRNAs with high $16 \mathrm{~S}$ identity to $E$. coli (96.2-99.6\%) had a minimal effect on sfGFP expression (Fig. 3c). However, replacement of intergenic sequences for moderately divergent O-rRNAs (81.5-96.2\%) substantially increased sfGFP expression (Fig. 3d). Interestingly, many nonfunctional O-rRNAs yielded robust sfGFP activities only after intergenic sequence replacement, namely $S$. marcescens, $V$. cholerae, $P$. aeruginosa, $A$. baumannii, and $B$. cenocepacia. Replacement of intergenic sequences for highly divergent O-rRNAs (69.8-82.3\%) failed to 
a
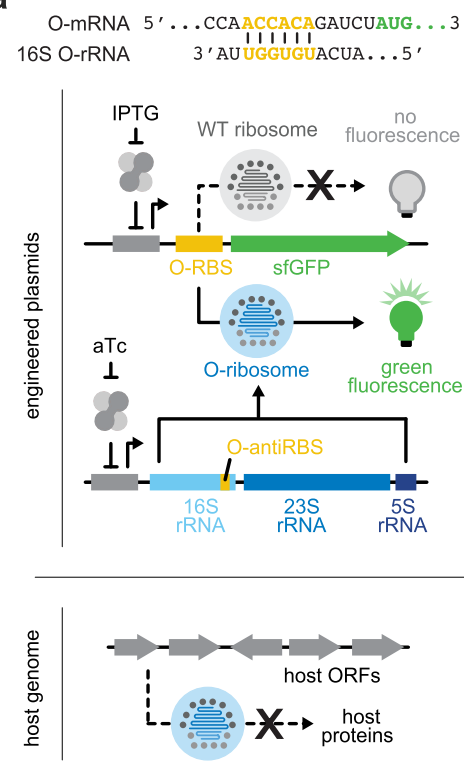

e

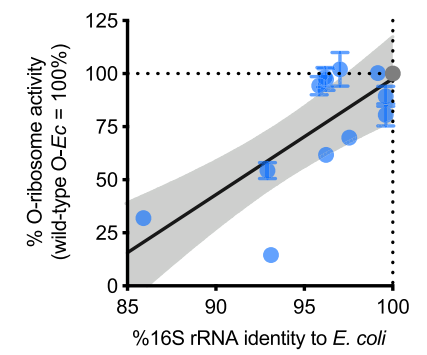

b

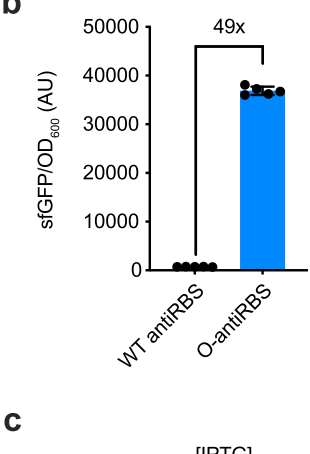

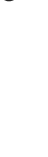

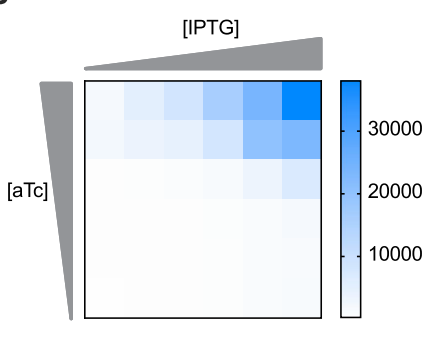

$\mathbf{f}$

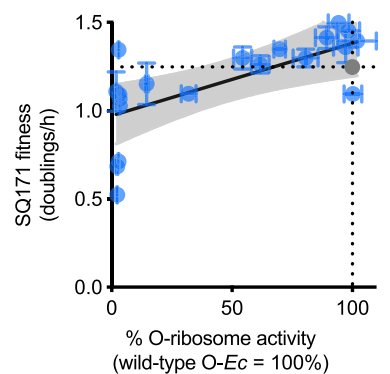

d

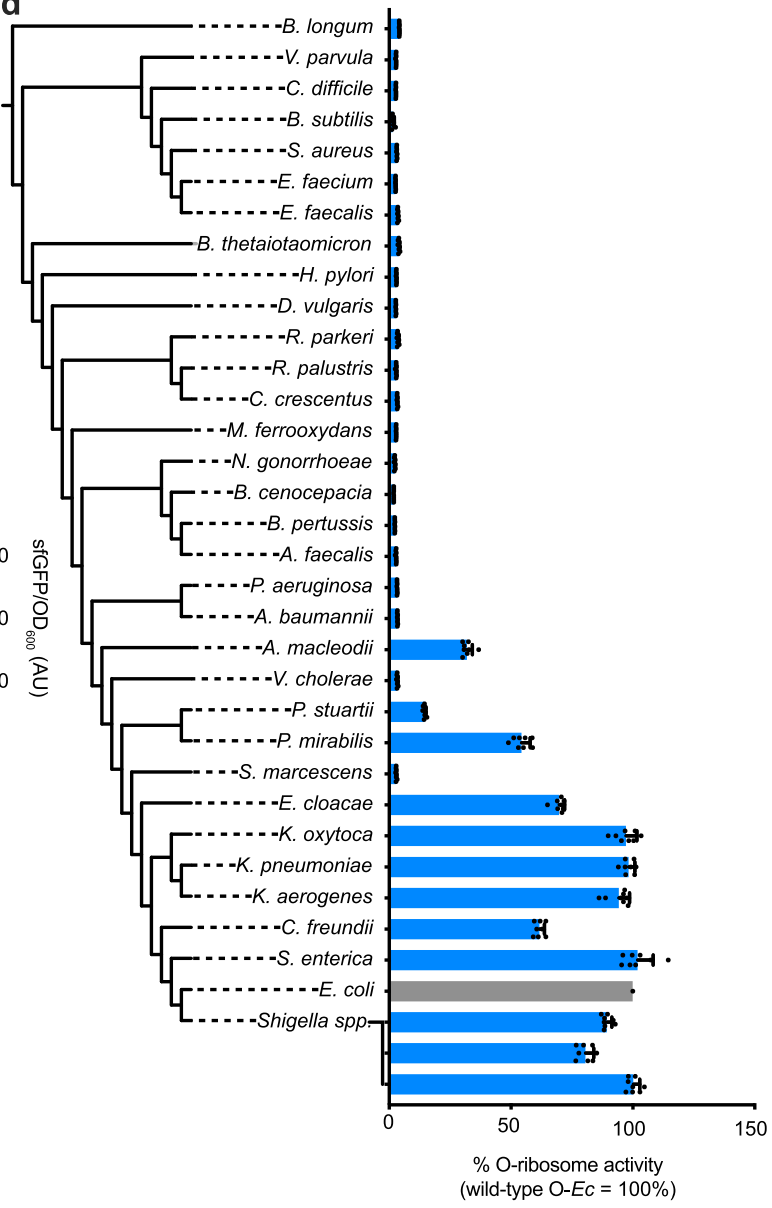

Fig. 2 Quantification of heterologous rRNA function using orthogonal translation. a Schematic representation of the orthogonal translation circuit. A superfolder GFP (sfGFP) reporter incorporates an orthogonal ribosome binding site (O-RBS) exclusively recognized by ribosomes that bear the complementary orthogonal anti-ribosome binding site (O-antiRBS), yielding a quantifiable fluorescent readout for heterologous ribosome activity. b Comparison of wild-type anti-ribosome binding site (WT antiRBS) and O-antiRBS E. coli ribosomes translating the O-RBS sfGFP reporter ( $n=5$ ). c Inducer dependence of the orthogonal translation circuit $(n=2)$. $\mathbf{d}$ Heterologous rRNA activities of 34 ribosomal RNA (rRNA) operons (blue columns) in addition to the E. coli O-rRNA control (gray column) as quantified by orthogonal translation $(n=8)$. e Correlation between $16 \mathrm{~S}$ rRNA sequence identity to E. coli (\%) and activity in the orthogonal translation genetic circuit (normalized to E. coli O-rRNA) for 15 functional heterologous O-rRNAs (mean activity $\geq 5 \%$ ), illustrating a correlation between orthogonal translation activity and $16 \mathrm{~S}$ identity $\left(99 \% \mathrm{Cl}, R^{2}=0.79\right)$. $\mathbf{f}$ Correlation between activity in the orthogonal translation circuit and fitness in SQ171 strain complementation assays for 21 heterologous rRNAs in addition to $E$. coli, illustrating a linear relationship ( $99 \% \mathrm{Cl}, R^{2}=0.47$ ). E. coli rRNA controls plotted in gray. Data reflect the mean and standard deviation of 1-8 biological replicates. Comprehensive SQ171 complementation and O-translation data (including precise number of replicates) reported in Supplementary Table 1. OD optical density, AU arbitrary units, wild-type O-Ec wild-type orthogonal E. coli rRNA, h hour. Source data are available in the Source Data File.

improve O-rRNA translation (Supplementary Fig. 4), suggesting that further engineering or supplementation with additional factors may be necessary to improve the activity of these highly divergent heterologous ribosomes. Finally, we reintroduced the wild-type antiRBS into the 21 engineered intergenic sequence-bearing rRNAs whose native counterparts were previously shown to support SQ171 survival, finding that SQ171 survival was maintained after intergenic sequence replacement, as was the relationship between SQ171 fitness and orthogonal translation activity (Fig. 3e). Taken together, these data suggest that rRNA processing may limit the assembly of more divergent heterologous rRNAs into functional ribosomes, and that engineering processing sites can considerably improve the activities of refractory heterologous ribosomes.

R-protein complementation further enhances heterologous ribosome activity. Highly divergent rRNAs $(<80 \% 16 \mathrm{~S}$ rRNA sequence identity to $E$. coli) failed to translate the orthogonal sfGFP transcript despite replacement of their intergenic sequences, suggesting that the formation of functional heterologous ribosomes may require supplementation with additional factors. As ribosomal proteins (r-proteins) are known to codiverge alongside their cognate rRNAs ${ }^{44,45}$, we hypothesized that E. coli r-proteins may only be capable of binding to and forming heterologous ribosomes with rRNAs sufficiently similar to $E$. coli. Complementing highly divergent rRNAs with cognate r-proteins would therefore be expected to improve heterologous ribosome activity.

In prokaryotes, r-proteins are typically encoded on five operons named $\alpha, \beta, s 10, s p c$, and str. R-proteins encoded within these five operons account for $\sim 60 \%(12 / 21$ SSU and 18/33 LSU in E. coli) of the full r-protein repertoire, ${ }^{36}$ with the remaining $\sim 40 \%$ distributed throughout the genome (Fig. 4a). Using $A$. baumannii O-rRNA bearing the E. coli intergenic sequences (30\% activity vs. E. coli O-rRNA), we analyzed potential improvements in activity when expressing the full set of 55 cognate r-proteins distributed through seven plasmids: five corresponding to the 
a

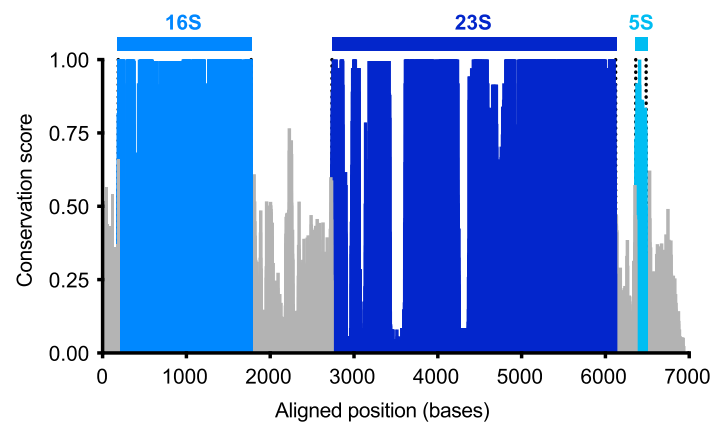

b

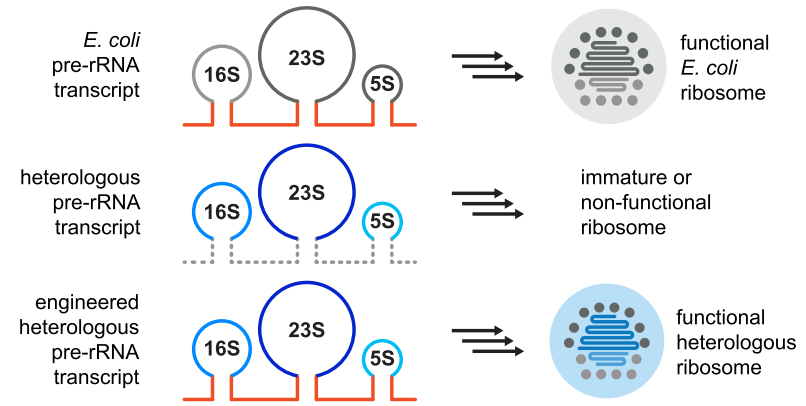

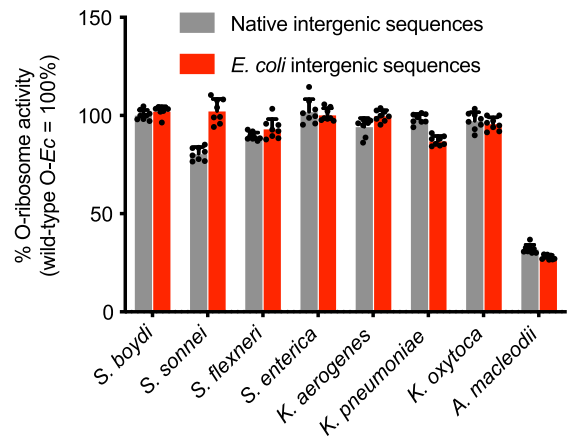

d

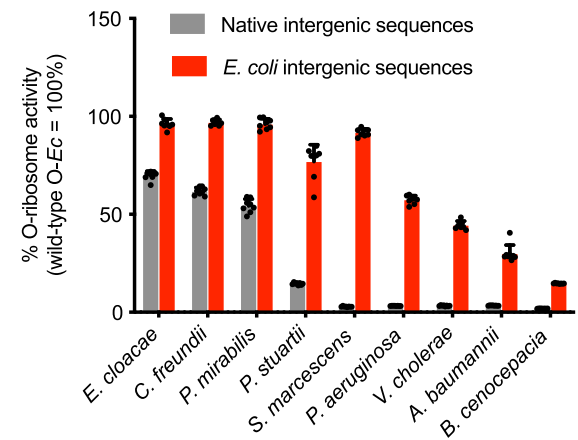

e

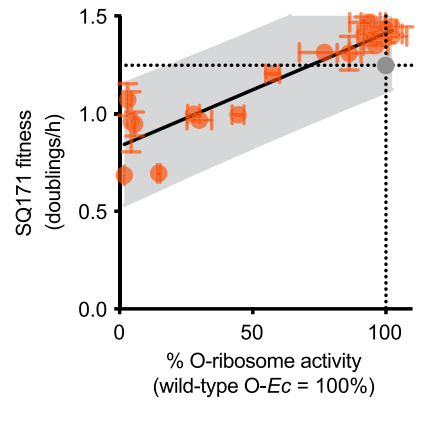

Fig. 3 Evaluating the effects of O-rRNA intergenic sequence replacement on heterologous translation. a Per-base sequence conservation ${ }^{41}$ across 34 evaluated rRNA operons, showing limited conservation in intergenic regions as compared to structural rRNA genes. b Schematic representation of the intergenic sequence replacement strategy. c Effects of intergenic sequence replacement on O-rRNAs with high $16 S$ rRNA sequence identity to $E$. coli (96.2-99.6\%), as well as A. macleodii (85.9\%), illustrating a minimal effect on orthogonal translation $(n=8)$. $\mathbf{d}$ Effects of intergenic sequence replacement on O-rRNAs with intermediate $16 \mathrm{~S}$ rRNA sequence identity to $E$. coli (81.5-97.4\%), illustrating a substantial effect on intergenic sequence replacement $(n=8)$. e Correlation between activity in the O-translation circuit and fitness in SQ171 strain complementation assays for 21 rRNAs evaluated after intergenic sequence replacement, showing a linear relationship. E. coli rRNA control plotted as a gray circle $\left(99 \% \mathrm{Cl}, R^{2}=0.84\right)$. Data reflect the mean and standard deviation of 3-8 biological replicates. Comprehensive SQ171 complementation and O-translation data (including precise number of replicates) reported in Supplementary Table 1. Wild-type O-Ec wild-type orthogonal E. coli rRNA, O-ribosome orthogonal ribosome, h hour. Source data are available in the Source Data File.

naturally occurring r-protein operons and two artificial operons (AOs) encoding the remaining r-proteins (Fig. 4a). To capture potential epistatic interactions involving either SSU or LSU rproteins, we enriched each artificial operon in either SSU $\left(\mathrm{AO}_{1}\right)$ or LSU $\left(\mathrm{AO}_{2}\right)$ r-proteins.

When tested alongside A. baumannii O-rRNA, only $A b \mathrm{AO}_{1}$ (comprising mostly SSU r-proteins) substantially improved sfGFP expression (Fig. 4b). Notably, complementation by a plasmid containing every A. baumannii SSU r-protein (S1-S21) yielded similar levels of activity as $A b \mathrm{AO}_{1}$, suggesting the latter contains all SSU r-proteins necessary to improve A. baumannii heterologous translation (Supplementary Fig. 5a). Copy-up mutations ${ }^{46}$ to $A b \mathrm{AO}_{1}$ further improved apparent activity of this heterologous ribosome, exceeding the activity level of the E. coli O-rRNA (Supplementary Fig. 5b). To identify specific $\mathrm{r}$-proteins responsible for this increase in heterologous ribosome activity, we sequentially deleted r-proteins from $A b \mathrm{AO}_{1}$ and found that robust sfGFP activity was maintained in all instances (Supplementary Fig. 5c), suggesting that one or more r-proteins were functionally redundant. Analysis of individual r-proteins confirmed this assessment, highlighting that expression of either $A b S 20$ or AbS16 improved A. baumannii heterologous Oribosome activity to levels comparable to the E. coli O-rRNA (Fig. 4c).

We note, however, that excessive protein overexpression alongside orthogonal translation circuits can have pleiotropic consequences on apparent translational activity. Using mCherry as a surrogate for cognate r-protein overexpression alongside
O-rRNA-dependent sfGFP production, we observe a characteristic isocost line that describes the production of two proteins under the constraints of a restricted metabolic budget (Supplementary Fig. 5d). ${ }^{47}$ Furthermore, O-rRNA promoter choice can dramatically affect orthogonal translation activity, as promoters with repetitive elements are rapidly recombined under high expression to mitigate the associated ribosome production burden (Supplementary Fig. 5e).

We extended this analysis to $A$. macleodii O-rRNA bearing the $E$. coli intergenic sequences $(17 \%$ activity vs. E. coli $\mathrm{O}-$ rRNA), finding again that only $A m \mathrm{AO}_{1}$ substantially improved sfGFP expression (Fig. 4d). As this finding suggested an overlap with $A$. baumannii r-proteins that improve heterologous ribosome function, we expressed $A m \mathrm{AO}_{1}$ constituent proteins alongside $A m S 20$ and $A m S 16$, finding that combinations of either $A m S 20+A m S 16+A m S 1$ or $A m S 20+A m S 16+A m S 15$ were sufficient to improve $A$. macleodii $O$-rRNA function to levels comparable with the E. coli O-rRNA (Fig. 4e). We observed a smaller but appreciable increase in apparent orthogonal translation activity using $\mathrm{AmAO}_{2}$ (enriched in LSU r-proteins) (Fig. 4d, Supplementary Fig. 6a, b). Dissecting the contributions $\mathrm{AmAO}_{2}$ r-proteins did not provide a comparable set of complementing r-proteins, as most genes contributed minor enhancements that collectively improved orthogonal translation activity (Supplementary Fig. 6b-e). However, these results confirmed that complementation with only a small number of cognate r-proteins can have substantial effects on heterologous ribosome function in E. coli. 
a

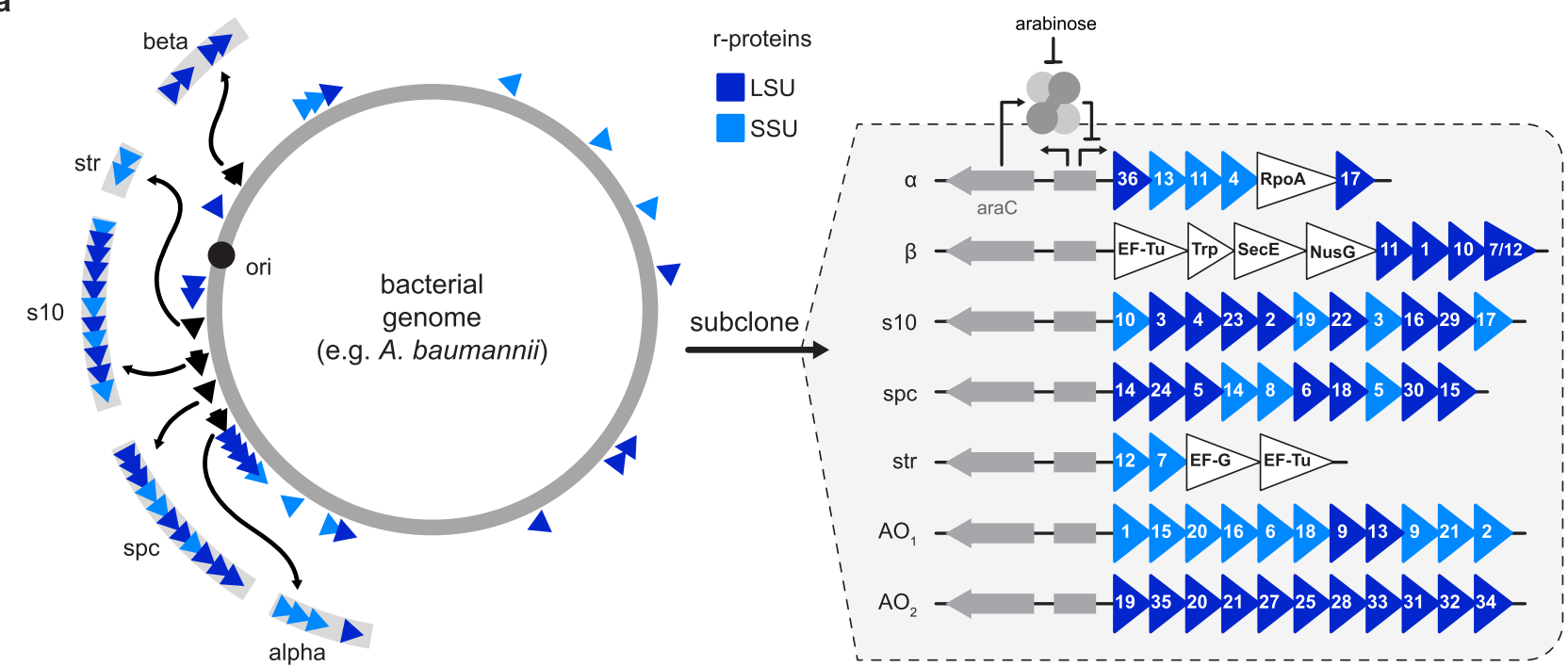

b

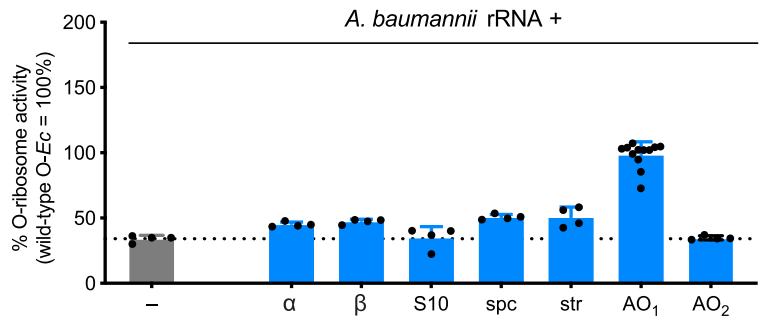

d

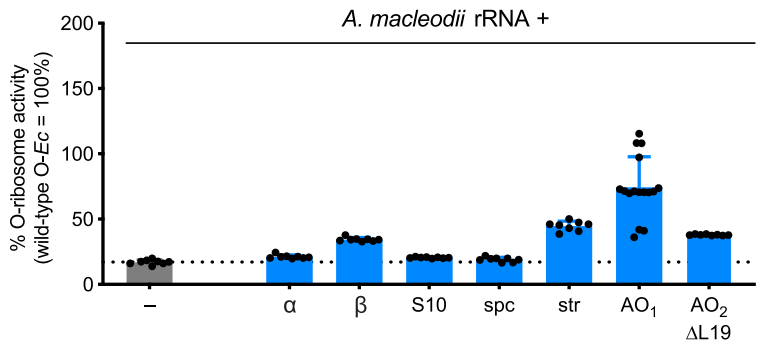

c

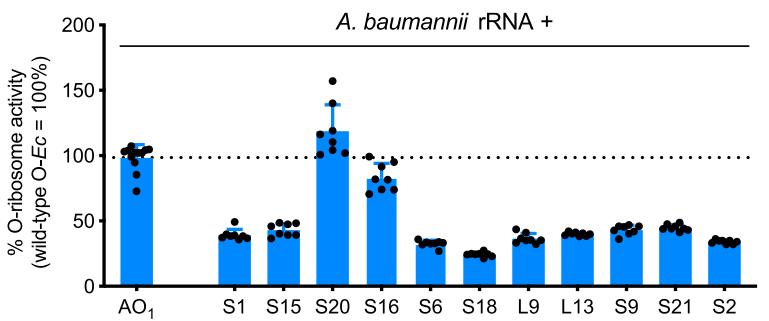

e

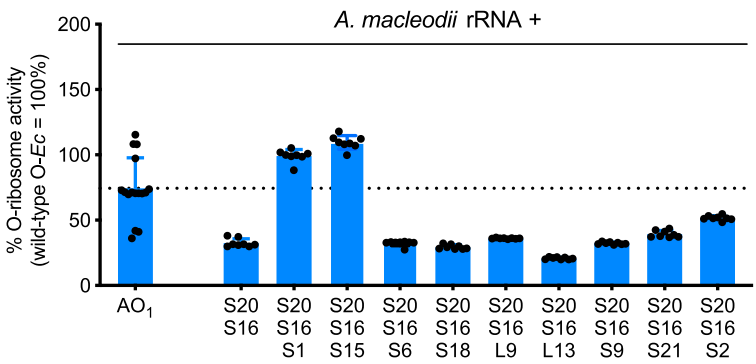

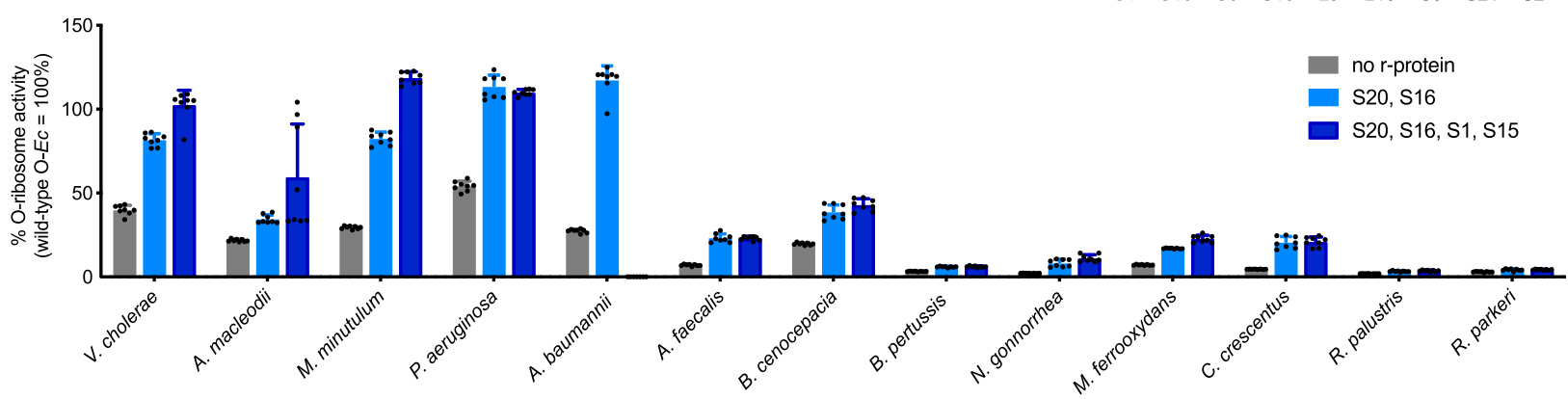

Fig. 4 Cognate r-proteins complementation improves heterologous O-rRNA activity. a Schematic representation of natural ribosomal protein ( $r$-protein) genomic organization for a given microbial genome and corresponding plasmid architecture for heterologous orthogonal ribosomal RNA (O-rRNA) complementation. b A. baumannii $\mathrm{AO}_{1}$ enhances cognate heterologous rRNA activity $\left(n=8\right.$ for $\mathrm{AO}_{1}$; otherwise $\left.n=4\right)$. c $A$. baumannii S20 and S16 enhance $A$. baumannii O-ribosome activity to levels comparable to $E$. coli O-ribosomes $\left(n=8\right.$ for $A_{1}$; otherwise $\left.n=4\right)$. d $A$. macleodii $A O_{1}$ similarly improves cognate heterologous rRNA activity $\left(n=16\right.$ for $\mathrm{AO}_{1}$; otherwise $\left.n=8\right) . \mathrm{AO}_{2}$ is expressed with $\mathrm{L} 19$ deleted due to observed toxicity (Supplementary Figs. 6a, b). e Cognate S1 or S15, alongside S20 and S16, maximize A. macleodii O-ribosome activity $\left(n=16\right.$ for AO ${ }_{1}$ otherwise $\left.n=8\right)$. f Cognate S20, S16, S1, and S15 supplementation alongside cognate heterologous O-rRNAs. Toxicity is observed when expressing the four proteins together in A. macleodii and A. baumannii (Supplementary Fig. 7a; $n=8$ ). Data reflect the mean and standard deviation of 4-16 biological replicates. Comprehensive O-translation data reported in Supplementary Table 1. Wild-type O-Ec wild-type orthogonal E. coli rRNA, SSU small subunit, LSU large subunit. Source data are available in the Source Data File. 
rRNA divergence predicts rules for cognate $\mathrm{R}$-protein complementation. The interface between rRNA and r-proteins is subject to extensive coevolution and divergence between related organisms ${ }^{44,45}$. Overlap between SSU r-protein complements that improved A. baumannii and $A$. macleodii O-rRNA activity suggested that the same r-proteins may improve the function of O-rRNAs derived from a variety of species. Indeed, the identified r-protein combinations improved activities of increasingly distant O-rRNAs: $P$. aeruginosa, $V$. cholerae, Marinospirillum minutulum, A. faecalis, B. cenocepacia, Neisseria gonorrhoeae, $M$. ferrooxydans, and Caulobacter crescentus (Fig. 4f).

Interestingly, this complete set of four r-proteins (S20, S16, S1, and S15) was not necessary for the observed improvement in activity for all evaluated O-rRNAs (Supplementary Fig. 7b, c). R-proteins S20 and S16 are functionally redundant when expressed alongside cognate O-rRNAs derived from species more phylogenetically related to $E$. coli: $V$. cholerae, A. macleodii, $M$. minutulum, $P$. aeruginosa, and B. cenocepacia. Uniquely, S16 has no effect on $A$. faecalis $O$-translation, where only S20 improves apparent activity. However, both proteins are necessary for enhanced activity when expressed alongside O-rRNAs derived from the more distant species $N$. gonorrheae, M. ferrooxydans, and C. crescentus (Supplementary Fig. 7b). Extending our analysis to the complete set of four proteins, we found that the addition of both $\mathrm{S} 1$ and $\mathrm{S} 15$ is necessary for maximal activities of $V$. cholerae and $M$. minutulum O-rRNAs, but neither r-protein has an effect when expressed alongside S20 and S16 for O-rRNAs derived from more divergent species (Supplementary Fig. 7c).

In order to determine r-proteins necessary to complement more divergent O-rRNAs, we extended our operon-based complementation approach to rRNAs derived from B. cenocepacia (betaproteobacteria; $81.5 \% 16 \mathrm{~S}$ rRNA sequence ID to E. coli), Rickettsia parkeri (alphaproteobacteria; 76.8\%), and Enterococcus faecalis (bacilli; 76.1\%). However, the only appreciable increase in orthogonal translation was observed in $B$. cenocepacia $\mathrm{AO}_{1}$, which we attributed to the contributions of S20 and S16 identified earlier (Figs. $4 \mathrm{f}$ and $5 \mathrm{a}-\mathrm{c}$ ). Having found sequence divergence from $E$. coli to be a powerful predictor of relevant features for heterologous rRNA supplementation, we manually identified 5 regions in the E. faecalis $16 \mathrm{~S}$ rRNA with particularly low sequence identity to $E$. coli via pairwise alignment (Supplementary Fig. 8$)^{48}$. As these divergent elements make extensive contacts with r-proteins in the E. coli ribosome (PDB: 4YBB $)^{49}$, divergence from $E$. coli in these sequences suggested an inability to efficiently bind to the requisite r-proteins.

To validate the functional relevance of these observations, we constructed variants of the E. coli O-rRNA in which these helices were replaced with their cognate E. faecalis helices, finding that orthogonal translation was abrogated in only 2 instances (transplantation of helices h9/h10 and h26; Fig. 5d). Seven rproteins would be expected to bind these helices based on existing ribosomal structures (EfS2, EfS8, EfS18, EfS12, EfS20, EfS16, and $E f S 17)^{49}$, and indeed supplementation with this set yielded a detectable increase in orthogonal translation activity (Fig. 5e). The deletion of EfS 8 and EfS18 from this set of proteins had no effect on activity, resulting in a set of 5 proteins that allowed $E$. faecalis O-rRNA activity to reach levels equivalent to $9.5 \%$ of the E. coli O-rRNA (Fig. 5e). Interestingly, this same set of 5 r-proteins was less effective than the combination of S20 and S16 for B. cenocepacia and M. ferrooxydans (81.5\% and $80.1 \% 16 \mathrm{~S}$ rRNA sequence identity to $E$. coli, respectively), but was more effective for the more distantly related $R$. parkeri $(76.8 \%)$ and $E$. faecalis $(76.1 \%)$ (Fig. 5f). We hypothesize that for O-rRNAs derived from more divergent organisms, the complete set of 5 r-proteins may be necessary to form a functional complex that cannot be formed by E. coli r-proteins. However, for O-rRNAs more related to $E$. coli, cognate r-proteins may compete with $E$. coli proteins for binding, forming less functional ribosomes. This finding highlights the importance of identifying the minimal subset of r-proteins necessary to improve function. Furthermore, we note that this set of $5 \mathrm{r}$-proteins is distributed across 2 naturally occurring operons in addition to the artificial operon $\mathrm{AO}_{1}$, obscuring these interactions from the 7-operon approach used above. Collectively, these results indicate that rRNA/ $\mathrm{r}$-protein codivergence can be used to predict r-protein repertoires that enhance the activity of heterologous ribosomes in E. coli.

Assessing exchange between $E$. coli and heterologous ribosome subunits. While our analysis provided guidelines to improve refractory heterologous ribosome function, the exclusive identification of SSU r-proteins suggested that cognate heterologous LSUs may be poorly active in E. coli. Instead, E. coli LSUs may interact with heterologous SSUs to enable orthogonal translation (Supplementary Fig. 10a). Conversely, many heterologous rRNAs support SQ171 strain survival, suggesting sufficient levels of activity by many heterologous LSUs (Figs. 1c, $2 \mathrm{f}$ and 3e, Supplementary Table 1).

To assess the degree of association between $E$. coli LSUs and heterologous SSUs, we developed an erythromycin-dependent reporter to distinguish between genome- (E. coli; erythromycinresistant) and episome-derived (heterologous; erythromycinsensitive) LSUs. We first generated the erythromycin-resistant strain S4246, wherein all seven genomic 23S genes $(\mathrm{rrlA}-\mathrm{H})$ of S2060 cells were mutated (A2058 $\left.{ }^{50}\right)$ to mitigate macrolide binding in the ribosomal exit tunnel (Fig. 6a) ${ }^{51,52}$. Next, we introduced the ErmC leader peptide, erm CL, ahead of the orthogonal sfGFP reporter, ensuring that reporter translation would be abrogated via erythromycin- and erm CL-dependent translational stalling ${ }^{53}$ (Fig. 6b).

To validate this sensor, we assessed the erythromycin sensitivity of an episome-derived E. coli orthogonal ribosome encoding or lacking the identical A2058U mutation. As a control, we leveraged a recently described stapled $E$. coli ribosome $(\mathrm{d} 2 \mathrm{~d} 8)^{7}$ that preferentially uses a covalently linked $23 \mathrm{~S}$ rRNA, with or without the A2058U mutation. Using this sensor/strain combination, we find that A2058U-LSUs show no appreciable change in orthogonal translation upon erythromycin dosing (Fig. 6c). Conversely, unmutated LSUs show a marked reduction in orthogonal translation in an erythromycin dose-dependent manner. Unstapled E. coli LSUs lacking the A2058U mutation re-sensitized S4246 cells to erythromycin whereas the stapled counterpart did not (Fig. 6d), suggesting that plasmid-encoded LSUs may co-assemble with genome-encoded SSUs to generate erythromycin-sensitive ribosomes incapable of translating essential E. coli genes (Supplementary Fig. 10b).

We extended this analysis to a set of 20 functional heterologous ribosomes. For heterologous ribosomes with high $16 \mathrm{~S}$ sequence identity to $E$. coli $(\geq 99.2 \%)$, we observe a dramatic reduction in both sfGFP translation and cell viability (Fig. 6e, Supplementary Fig. 10c, Supplementary Table 3), reflecting appreciable exchange between host and heterologous ribosomes. Interestingly, heterologous ribosomes bearing intermediate homology (92.9-97.0\%) showed extensive reduction in sfGFP signal upon erythromycin treatment with no associated viability defect. These reductions in sfGFP signal were comparable to or greater than the corresponding effect on the $\mathrm{d} 2 \mathrm{~d} 8$ stapled $E$. coli ribosome, suggesting a similar degree of association between cognate subunits. To ensure that these findings were not an artifact of heterologous ribosomes' differential antibiotic susceptibilities, we verified their erythromycin sensitivities with and without the A2058U resistance- 
a

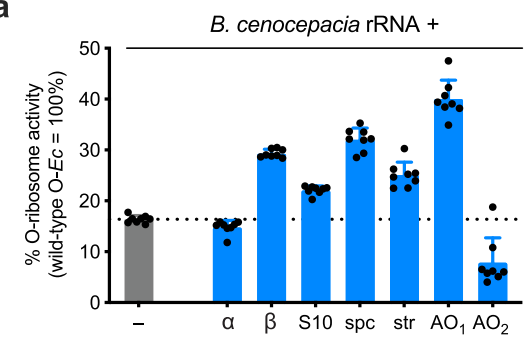

b

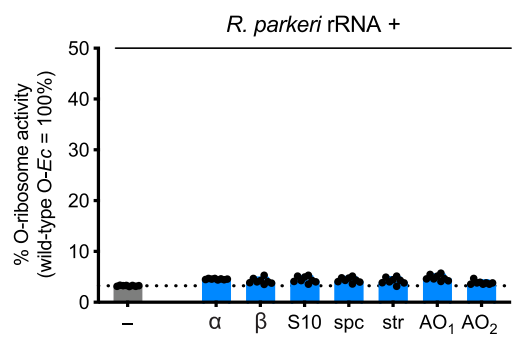

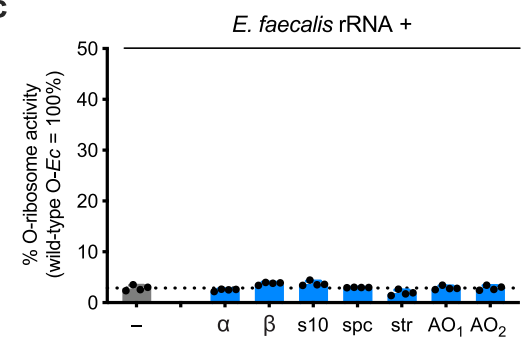

d

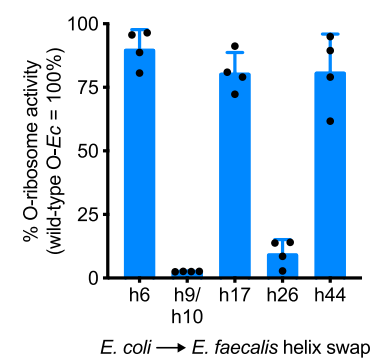

e

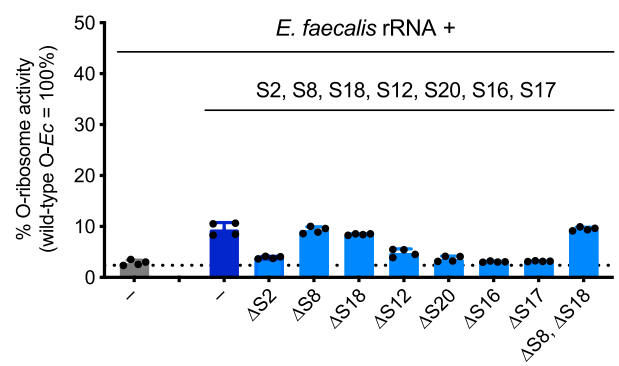

f

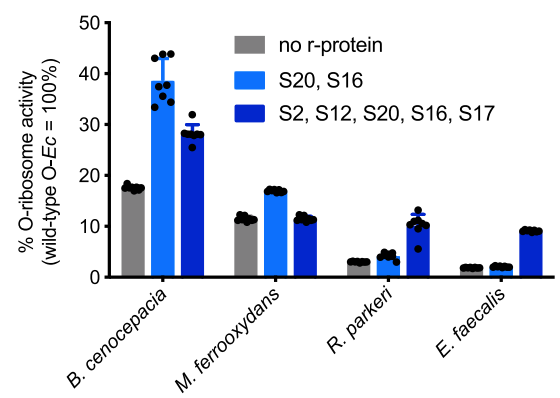

Fig. 5 Phylogenetically guided determination of cognate r-proteins that improve highly divergent heterologous O-ribosomes in E. coli. a-c No single contiguous operon substantially improves translation activity of O-rRNAs derived from a B. cenocepacia $(n=8), \mathbf{b} R$. parkeri $(n=8)$, or $\mathbf{c} E$. faecalis $(n=4)$. d Only 2 of the identified 5 regions with high sequence divergence between E. coli and E. faecalis $16 \mathrm{~S}$ rRNAs abrogate E. coli O-translation when replaced with cognate $E$. faecalis sequences $(n=4)$. e Cognate S2, S8, S18, S12, S20, S16, and S17 (which directly contact h9, h10, and h26 49) result in an increase in E. faecalis O-rRNA translation. Further analysis showed that S8 and S18 are not required for this increase $(n=4)$. f R-proteins S2, S12, S20, S16, and S17 collectively improve cognate O-rRNA translation for highly divergent species, but are not as effective as S20 and S16 alone for less divergent species. $E$. faecalis cognate ribosomal proteins ( $r$-proteins) are expressed from a low copy number backbone (WT RepA SC101 origin) to limit toxicity ( $n=8$ ). Data reflect the mean and standard deviation of 4-8 biological replicates. Comprehensive O-translation data reported in Supplementary Table 1. Wild-type O-Ec wild-type orthogonal E. coli, O-ribosome orthogonal ribosome, h helix. Source data are available in the Source Data File.

conferring mutation in SQ171 cells. For all tested heterologous ribosomes, erythromycin sensitivity was nearly identical to that of E. coli (Supplementary Fig. 9, Supplementary Table 2). Finally, for more divergent heterologous ribosomes (79.3-90.3\%), sfGFP signal decreased only minimally upon erythromycin treatment. This signal may reflect translation using the E. coli LSU, where heterologous LSUs derived from highly divergent species may be poorly assembled in E. coli. Overall, this suggests that additional rRNA operon modifications or complementation with cognate factors may be necessary to enable the preferential usage of the heterologous LSU.

In an attempt to reduce association between heterologous and $E$. coli ribosomal subunits, we extended the above rRNA stapling approach $^{7}$ to the same 20 heterologous ribosomes. This staple appears to be extensible to rRNAs with high sequence identity $(\geq 99.2 \% 16 \mathrm{~S})$ to E. coli (Supplementary Fig. 10d-f). However, for most rRNAs, this approach does not increase erythromycin sensitivity. Hypothesizing that the $\mathrm{d} 2 \mathrm{~d} 8$ linkers were not suited to heterologous LSUs, we therefore generated "hybrid" ribosomes comprising heterologous SSUs stapled to E. coli LSUs. Finding that these hybrid ribosomes vary considerably in erythromycin sensitivity, we postulate that the rRNA linker would require independent optimization for each heterologous ribosome. Collectively, these data suggest that intermediately divergent heterologous SSUs may preferentially associate with the cognate LSUs in E. coli, and could serve as starting points for future engineering efforts.

\section{Discussion}

We constructed a library of 34 heterologous ribosomes derived from species across a broad phylogenetic range and expressed in E. coli. We assayed the functionality of these ribosomes using both $\Delta 7$ strain complementation and orthogonal translation, and found a high degree of correlation between the two assays. Replacement of intergenic sequences with those of $E$. coli, as well as supplementation with only a small subset of r-proteins (S20, S16, S1, and S15), improved expression from orthogonal heterologous rRNAs. While O-rRNAs with high sequence identity (as little as $96.2 \%$ 16S rRNA sequence identity to $E$. coli) natively translated sfGFP at robust levels, substitution of intergenic sequences allowed for O-rRNAs as divergent as $P$. mirabilis (92.9\%) to translate the orthogonal transcript at levels similar to the E. coli O-rRNA. Supplementation with r-proteins S20 and S16 allowed for similarly robust levels of translation from O-rRNA derived from A. baumannii (84.3\%). Using a more extensive set of r-proteins, we were able to observe heterologous translation from O-rRNAs as diverged as E. faecalis (76.1\%). Finally, using an erythromycin-dependent reporter, we found that a subset of heterologous SSUs appeared to preferentially associate with their cognate LSUs.

Collectively, our findings establish orthogonal translation as a viable alternative to $\Delta 7$ complementation for evaluating the function of heterologous rRNAs and suggest generalizable strategies for enhancing heterologous rRNA function. Interestingly, of the four $r$ proteins found to be broadly important for O-rRNA function, only two (S1 and S16) have been found to be essential for viability in $E$. coli via gene knockout, ${ }^{54,55}$ suggesting that essentiality cannot serve as a predictor of crucial factors enhancing O-rRNA function. We therefore sought to determine whether we could derive rules for predicting $\mathrm{r}$-proteins necessary for complementing heterologous $\mathrm{O}$ rRNAs. Using sequence similarities of heterologous SSU r-proteins to their E. coli homologues, we found that r-proteins empirically discovered to be crucial for complementation (S20, S16, and S15) tend to be those with the lowest sequence similarity (Supplementary Fig. 11a-n). Particularly in the case of S20 and S16, this may reflect their roles as primary binders to the $\mathrm{RRNA}^{56}$, which are 
a

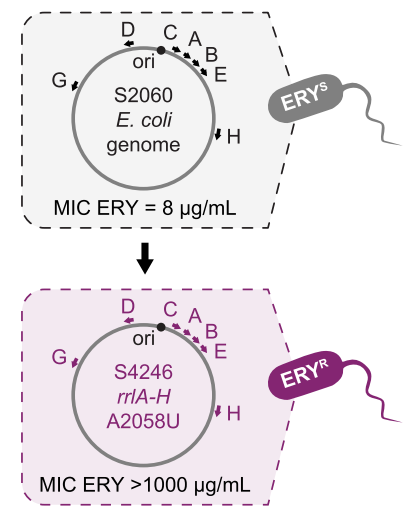

b

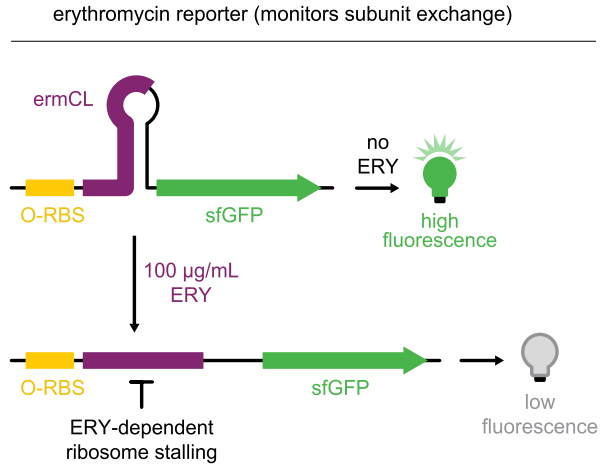

$\mathbf{e}$
C

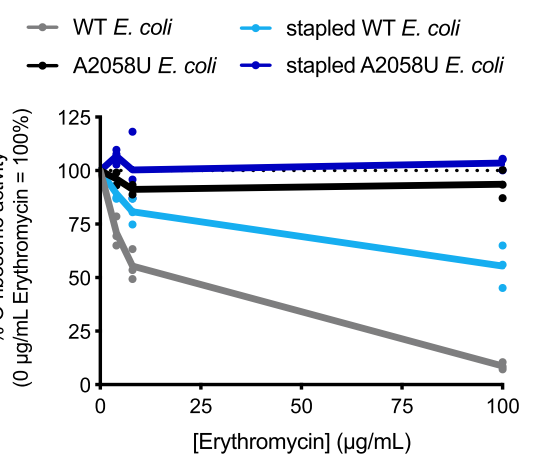

d

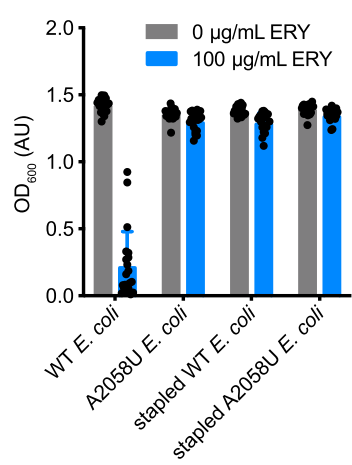

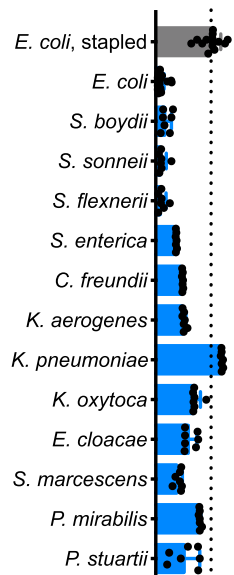

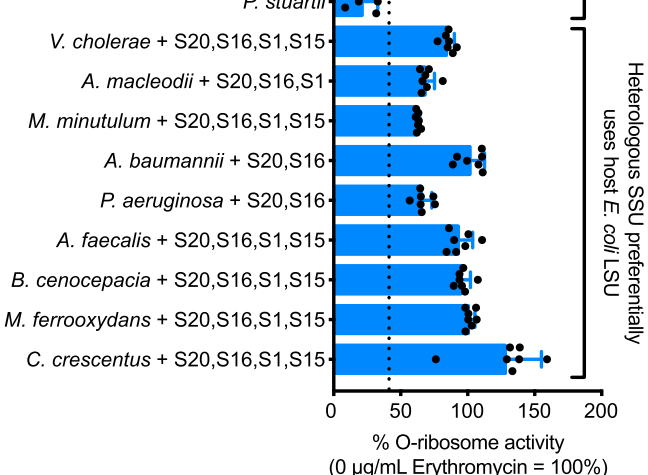

Fig. 6 The erythromycin-dependent orthogonal translation reporter system. a Creation of the erythromycin (ERY)-resistant E. coli strain S4246. All 7 rrl $(A-H) 23$ S ribosomal RNA ( $r R N A$ ) genes were mutated (A2058U) via oligonucleotide recombineering to endow high erythromycin resistance (ERY; MIC > $1000 \mu \mathrm{g} \mathrm{mL}{ }^{-1}$ ). b Schematic representation of the ERY-dependent superfolder GFP (sfGFP) reporter. In the absence of ERY, sfGFP is efficiently translated via orthogonal translation. Addition of ERY $\left(100 \mu \mathrm{gL}^{-1}\right)$ promotes translation stalling at the ermC leader peptide (ermCL), abrogating sfGFP translation by ERY-sensitive large subunits (LSUs). c Free and stapled ERY-sensitive LSUs show a marked reduction in sfGFP production at high inhibitor concentrations, whereas the corresponding ERY-resistant (23S A2058U) LSUs show no appreciable change in activity ( $n=2$ for A2058U E. coli at $4 \mu \mathrm{g} / \mathrm{mL}$ ERY; otherwise $n=3$ ). d ERY-sensitive LSUs re-establish strain sensitivity to ERY due to free subunit exchange between episomally and genomically derived ribosomes $(n=21)$. e Evaluation of intersubunit exchange using the ERY-dependent reporter system. Heterologous ribosomes with high 165 sequence identity to $E$. coli $(\geq 99.2 \%)$ appear to freely exchange with host subunits, while heterologous ribosomes with intermediate sequence identity (97.0-92.9\%) preferentially associate with cognate subunits at a rate comparable to the stapled E. coli ribosome. More divergent heterologous ribosomes (90.3-79.3\%) preferentially utilize E. coli large subunits ( $n=28$ for $E$. coli and $n=14$ for $E$. coli, stapled; otherwise $n=7$ ). Data for each ribosome is normalized to its corresponding sfGFP signal at $0 \mu \mathrm{g} \mathrm{mL}-1$ ERY. Data reflect the mean and standard deviation of the indicated biological replicates. Comprehensive data reported in Supplementary Table 3. MIC = minimum inhibitory concentration; OD optical density, O-RBS orthogonal RBS, O-ribosome orthogonal ribosome, SSU small subunit. Source data are available in the Source Data File.

known to diverge more rapidly. ${ }^{44,45}$ All three proteins, however, play important roles in the earliest stages of $30 \mathrm{~S}$ assembly. ${ }^{56}$ Similarly, S1 often interacts with mRNAs in proximity to the RBS during translational initiation, ${ }^{57,58}$ suggesting that it may play a role in mediating correct RBS/antiRBS interactions using noncanonical (orthogonal) pairs.

In the future, it may be possible that heterologous rRNAs, specifically those of relevance to human health, can be expressed in E. coli for the high-throughput discovery of ribosome-targeting antibiotics. More broadly, heterologous ribosomes and strategies described here may serve as alternative starting points for the discovery and laboratory evolution of novel translational properties. Through the use of such ribosomes, synthetic biologists can take advantage of the myriad functionalities that natural Darwinian evolution has generated in phylogenetically diverse bacterial ribosomes.

\section{Methods}

General methods. Unless otherwise noted, all PCRs were performed using Phusion U HotStart DNA Polymerase (Life Technologies). Water was purified using a MilliQ water purification system (MilliporeSigma). Antibiotics (Gold
Biotechnology) were used at the following concentrations for plasmid selection, unless otherwise noted: $30 \mu \mathrm{g} \mathrm{mL}^{-1}$ kanamycin, $40 \mu \mathrm{g} \mathrm{mL}{ }^{-1}$ chloramphenicol, 50 $\mu \mathrm{g} \mathrm{mL}^{-1}$ carbenicillin, $100 \mu \mathrm{g} \mathrm{mL}^{-1}$ spectinomycin. Antibiotics were used at onethird concentrations for strains bearing three unique plasmids. Unless otherwise noted, all DNA manipulations were performed in NEB Turbo cells (New England Biolabs) or Mach1F cells (Mach1 $\mathrm{T}^{\mathrm{R}}$ cells (Thermo Fisher Scientific) mated with F' episome of the previously described $S 2060^{59}$ strain). All fluorescence and luminescence assays were carried out using E. coli $\mathrm{S} 2060^{59}$.

Chemically competent cell preparation. Chemically competent cells were used for all cloning and assay pipelines. A glycerol stock of the appropriate strain was used to start a $2 \mathrm{~mL}$ culture supplemented with the appropriate antibiotics and grown up overnight at $30^{\circ} \mathrm{C}$ at $300 \mathrm{RPM}$. The saturated culture was diluted 1:1000 in $50 \mathrm{~mL} 2 \mathrm{xYT}$ (United States Biological) with appropriate antibiotics and grown to $\mathrm{OD}_{600}=0.3-0.5$ in a $37^{\circ} \mathrm{C}$ shaker at $300 \mathrm{RPM}$. Cells were pelleted in a pre-chilled conical tube (VWR) by centrifugation at $8000 \mathrm{~g}$ for $10 \mathrm{~min}$ at $4{ }^{\circ} \mathrm{C}$. The supernatant was removed and the cells were resuspended in approximately $20 \mathrm{~mL} 10 \%$ glycerol, then pelleted by centrifugation at $8000 \mathrm{~g}$ for $10 \mathrm{~min}$ at $4{ }^{\circ} \mathrm{C}$. The supernatant was removed and the cells were resuspended in chilled TSS buffer (2xYT media supplemented with 5\% DMSO, 10\% PEG2250, $2 \mathrm{mM} \mathrm{MgCl} 2$ ). Cells were flash frozen in liquid $\mathrm{N}_{2}$ at $100 \mu \mathrm{L}$ aliquots and transferred to $-80{ }^{\circ} \mathrm{C}$ storage.

USER cloning. Plasmids were constructed using USER cloning, or a combination of USER cloning and overlap extension PCR. In USER cloning, primers are 
designed to include a deoxyuracil base approximately $10-20$ bases from the $5^{\prime}$ end of the primer; the region between the deoxyuracil base and the $5^{\prime}$ end of the primer is known as the "USER junction" and specifies the homology necessary for plasmid assembly. USER junctions were designed to have a $42^{\circ} \mathrm{C}<T_{\mathrm{m}}<70^{\circ} \mathrm{C}$, minimal secondary structure, and begin with a $\mathrm{dA}$ and end with a $\mathrm{dT}$ (the latter is replaced with a dU to act as a substrate for uracil DNA glycosylase during assembly). PCR products were gel purified using QIAquick Gel Extraction kit (Qiagen) and eluted to a final volume of $10 \mu \mathrm{L}$. Fragments were quantified using a NanoDrop 1000 Spectrophotometer (Thermo Fisher Scientific). For assembly, PCR products with complementary USER junctions were added in an equimolar ratio (0.1-1 pmol each) in a $10 \mu \mathrm{L}$ reaction containing 0.75 units DpnI (New England Biolabs), 0.75 units USER (Uracil-Specific Excision Reagent; Endonuclease VIII and Uracil-DNA Glycosylase) enzyme (New England Biolabs), 1 unit of CutSmart Buffer (50 mM potassium acetate, $20 \mathrm{mM}$ Tris-acetate, $10 \mathrm{mM}$ magnesium acetate, $100 \mu \mathrm{g} \mathrm{mL}^{-1}$ BSA at pH 7.9; New England Biolabs). Reactions were incubated at $37^{\circ} \mathrm{C}$ for $20 \mathrm{~min}$, then heated at $80{ }^{\circ} \mathrm{C}$ and slowly cooled to $12{ }^{\circ} \mathrm{C}$ at $0.1{ }^{\circ} \mathrm{C} / \mathrm{s}$ in a thermocycler. Inserts of plasmids " $\mathrm{AO}_{1}$ ", " $\mathrm{AO}_{2}$ ", and "S1-S21," consisting of many small fragments, were cloned using overlap extension PCR. Primers were designed containing $\sim 15$ bp overhangs complementary to the adjoining fragment. Individual fragments were amplified and gel purified as above, then 0.2 picomoles of each fragment was used in a $200 \mu \mathrm{L}$ PCR reaction to join each fragment together. This fragment was gel purified and USER assembly was used for cloning into the appropriate plasmid backbone. Assembled constructs were heat-shocked into chemically competent NEB Turbo or Mach1F cells: $100 \mu \mathrm{L} 2$ x KCM $(100 \mathrm{mM} \mathrm{KCl}$, $30 \mathrm{mM} \mathrm{CaCl}, 50 \mathrm{mM} \mathrm{MgCl}$ in MilliQ $\mathrm{H}_{2} \mathrm{O}$ ) was added to $100 \mu \mathrm{L}$ cells alongside plasmid DNA. Cells were incubated on ice for $15 \mathrm{~min}$, heat shocked at $42{ }^{\circ} \mathrm{C}$ for $2 \mathrm{~min}$, and placed back on ice for $2 \mathrm{~min}$. Cells were recovered in $1 \mathrm{~mL} 2 \mathrm{xYT}$ media at $37^{\circ} \mathrm{C}$ with shaking at $300 \mathrm{RPM}$ for a minimum of $45 \mathrm{~min}$. Cells were streaked on $1.8 \%$ agar-2xYT plates supplemented with the appropriate antibiotic(s).

Amplification of ribosomal operons and R-proteins. Ribosomal DNA, contiguous r-protein operons, and single r-protein ORFs were amplified from bacterial strains or the corresponding gDNA. Direct amplification from bacterial strains required boiling at $95{ }^{\circ} \mathrm{C}$ for $10 \mathrm{~min}$ in MilliQ water prior to PCR for efficien amplification. In cases where a non-type strain was used, universal primers (Supplementary Table 4 ) were used to amplify a $\sim 900 \mathrm{bp}$ fragment from the bacterial genome to include a partial $16 \mathrm{~S}$ element for subcloning and sequencing, allowing for closest sequenced genome determination. For species with high sequence variability between ribosomal operons, a representative operon was chosen based on maximal sequence homology to $E$. coli.

Bacterial strain genomic modifications. The erythromycin-resistant strain S4246 was generated using conventional recombineering 51,52 . Briefly, chemically competent S2060 cells were transformed with pKD46 $6^{52}$ and plated on 2xYT agar plates supplemented with $50 \mu \mathrm{g} \mathrm{mL}-1$ carbencillin at $30^{\circ} \mathrm{C}$. A single colony was picked, grown at $30^{\circ} \mathrm{C}$ in $2 \times Y T$ liquid medium supplemented with $50 \mu \mathrm{g} \mathrm{mL}-1$ carbencillin and $10 \mathrm{mM}$ arabinose, and made chemically competent when the culture reached the appropriate $\mathrm{OD}_{600}$. Chemically competent $\mathrm{S} 2060 / \mathrm{pKD} 46$ cells were transformed with the phosphothiorated recombineering oligonucleotide AB5708 (Supplementary Table 4) to introduce the $r r l A-H$ A2058U mutation on replichore $2^{51}$. Following recovery for $3 \mathrm{~h}$ at $30^{\circ} \mathrm{C}$, transformed cells were plated on $2 \mathrm{xYT}$ agar plates supplemented with $1000 \mu \mathrm{g} \mathrm{mL}-1$ erythromycin and incubated at $37^{\circ} \mathrm{C}$ to cure the resident $\mathrm{pKD} 46$ plasmid. Following overnight growth, single colonies were picked into 2xYT liquid medium supplemented with $50 \mu \mathrm{g} \mathrm{mL} \mathrm{m}^{-1}$ streptomycin, $10 \mu \mathrm{g} \mathrm{mL}^{-1}$ tetracycline, and $1000 \mu \mathrm{g} \mathrm{mL}^{-1}$ erythromycin and allowed to grow overnight at $37^{\circ} \mathrm{C}$. To assess the degree of $r r l A-H$ mutagenesis, cultures were used as PCR templates using primers AB5710 and AB5711 (Supplementary Table 4), and the PCR products were treated with the endonuclease HpyCH4III (New England Biolabs) according to the manufacturer's guidelines. Wild-type $r r l A-H$ genotypes show no digestion under these conditions, whereas complete conversion results in complete PCR product digestion. Intermediate (incomplete) digestion indicated in incomplete conversion of all seven genomic alleles. The completely converted strain S4246 was confirmed to be sensitive to the following antibiotics (ensures no resistance crosstalk with plasmid-borne markers): carbenicillin $(50 \mu \mathrm{g}$ $\left.\mathrm{mL}^{-1}\right)$, spectinomycin $\left(100 \mu \mathrm{g} \mathrm{mL}^{-1}\right)$, chloramphenicol $\left(40 \mu \mathrm{g} \mathrm{mL}^{-1}\right)$, and kanamycin $\left(30 \mu \mathrm{g} \mathrm{mL}^{-1}\right)$. The strain was confirmed to be resistant to the following antibiotics: streptomycin $\left(50 \mu \mathrm{g} \mathrm{mL}^{-1}\right)$, tetracycline $\left(10 \mu \mathrm{g} \mathrm{mL}^{-1}\right)$, and erythromcyin $\left(1000 \mu \mathrm{g} \mathrm{mL}^{-1}\right)$.

Fluorescence assays. For orthogonal translation assays, S2060 chemically competent cells were transformed with the E. coli O-rRNA plasmid and the relevant orthogonal reporter plasmid. Transformants were streaked on $1.8 \%$ agar-2xYT plates supplemented with kanamycin and carbenicillin. Plates were grown in a $37^{\circ} \mathrm{C}$ incubator for $16 \mathrm{~h}$. Colonies were picked into $500 \mu \mathrm{L}$ DRM (United States Biological) ${ }^{60}$ (supplemented with kanamycin, carbenicillin, $1 \mathrm{mM} \mathrm{IPTG}+/-1000 \mathrm{ng} \mathrm{mL}^{-1} \mathrm{aTc}$ ) in deepwell plates (VWR) and grown at $37^{\circ} \mathrm{C}$ with shaking at $900 \mathrm{RPM}$ for 20 hours. To assay heterologous O-rRNA function, chemically competent cells carrying the sfGFP reporter plasmid were prepared (S2060.sfGFP) and transformed with the appropriate O-rRNA plasmid. E. coli O-rRNA was always transformed alongside experimental O-rRNAs as a positive control. Transformants were streaked out and picked into media as above.

To assay r-protein effects on heterologous O-rRNA function, S2060.sfGFP chemically competent cells were co-transformed with the appropriate O-rRNA plasmid and r-protein plasmid. As a positive control, E. coli O-rRNA was transformed alongside an mCherry expression plasmid. In the absence of r-protein supplementation, heterologous O-rRNAs were transformed with mCherry to maintain consistent growth rates and antibiotic selection markers. Transformants were streaked on $1.8 \%$ agar-2xYT plates supplemented with kanamycin, carbenicillin, chloramphenicol, and $200 \mathrm{mM}$ glucose, picked into DRM supplemented with kanamycin, carbenicillin, chloramphenicol, $1 \mathrm{mM} \mathrm{IPTG,}$ $1000 \mathrm{ng} \mathrm{mL}^{-1} \mathrm{aTc},+/-10 \mathrm{mM}$ arabinose, and grown up as above. For assays using the erythromycin-dependent reporter, DRM was supplemented with $100 \mu \mathrm{g} \mathrm{mL}$ erythromycin in addition to the above inducers and antibiotics.

To quantify fluorescence output, $150 \mu \mathrm{L}$ of each culture were aliquoted into a 96-well black wall, clear bottom plate (Costar). $\mathrm{OD}_{600}$ and the appropriate excitation and emission wavelengths were used for fluorescence measurements (Supplementary Table 5) using either a SpectraMax M3 (Molecular Devices) or Spark (Tecan) plate reader running SoftMax Pro v6.4 or SparkControl v2.3, respectively. Fluorescence was normalized to $\mathrm{OD}_{600}$ after blank media subtraction. Data were normalized to E. coli O-rRNA sfGFP/OD 600 and expressed as a percentage. When assaying the effects of $r$-protein complementation, data were normalized to E. coli O-rRNA (sfGFP signal) upon mCherry control plasmid induction.

SQ171 cell viability assay. Chemically competent SQ171 18,35 cells were transformed with heterologous rRNAs as described above and recovered for up to $7 \mathrm{~h}$ in $2 \mathrm{xYT}$ in a $37^{\circ} \mathrm{C}$ shaker. The recovery culture was centrifuged at 10,000 RCF for $2 \mathrm{~min}$, then the pellet was resuspended in $100 \mu \mathrm{L}$ MilliQ water. The resuspended cells were diluted serially in seven, 10 -fold increments to yield eight total samples (undiluted, $10^{1-}, 10^{2}-, 10^{3}-, 10^{4}-, 10^{5}-, 10^{6}-$, and $10^{7}$-fold diluted). To determine the efficiencies of EP transformation and counter-selectable plasmid curing, $3 \mu \mathrm{l}$ of diluted cells were plated on $1.8 \%$ agar-2xYT plates (United States Biological) supplemented with spectinomycin $\left(100 \mu \mathrm{g} \mathrm{mL}^{-1}\right)$ and carbenicillin $\left(50 \mu \mathrm{gL}^{-1}\right)$, with or without $5 \%$ sucrose (Millipore Sigma). For picking single colonies, the remaining undiluted cells were plated on $1.8 \%$ agar-2xYT plates (United States Biological) containing spectinomycin $(100 \mu \mathrm{g} \mathrm{mL}-1)$, carbenicillin $\left(50 \mu \mathrm{g} \mathrm{mL} L^{-1}\right)$, and $5 \%$ sucrose. All plates were grown for $16-120 \mathrm{~h}$ in a $37^{\circ} \mathrm{C}$ incubator.

For erythromycin sensitivity assays, colonies transformed with the appropriate rRNA EP and surviving sucrose selection were picked and grown in DRM containing spectinomycin $\left(100 \mu \mathrm{g} \mathrm{mL}^{-1}\right)$, carbenicillin $\left(50 \mu \mathrm{g} \mathrm{mL}^{-1}\right)$, and $5 \%$ sucrose, with or without kanamycin $\left(30 \mu \mathrm{g} \mathrm{mL}^{-1}\right)$. Following overnight growth, cultures were diluted 100-fold into fresh DRM containing spectinomycin (100 $\mu \mathrm{g}$ $\left.\mathrm{mL}^{-1}\right)$ and carbenicillin $\left(50 \mu \mathrm{gL}^{-1}\right)$. Wild-type strains were evaluated at 12 erythromycin concentrations from $1000-0.002 \mu \mathrm{g} \mathrm{mL}^{-1}$ in 3-fold dilution increments, and A2058U-23S strains were evaluated from $2000-1 \mu \mathrm{g} \mathrm{mL}-1$ in two-fold dilution increments. Colonies that survived selection in kanamycin were excluded from final analysis, as survival in kanamycin indicates persistence of the resident pCSacB plasmid (which carries a KanR resistance cassette). Following overnight growth, $200 \mu \mathrm{L}$ of each culture were aliquoted into a 96-well black wall, clear bottom plate (Costar). $\mathrm{OD}_{600}$ was measured using a Spark (Tecan) plate reader. $\mathrm{IC}_{50}$ curves were fit using GraphPad Prism version 8

For all other SQ171 assays, colonies transformed with the appropriate EP and surviving sucrose selection were picked and grown in DRM containing spectinomycin $\left(100 \mu \mathrm{g} \mathrm{mL}^{-1}\right)$, carbenicillin $\left(50 \mu \mathrm{g} \mathrm{mL}^{-1}\right)$, and $5 \%$ sucrose. Following growth of the EP-carrying strains for up to 3 days, cultures were glycerol stocked. Overnight cultures were started from these glycerol stocks in DRM containing spectinomycin $\left(100 \mu \mathrm{g} \mathrm{mL}^{-1}\right)$, carbenicillin $\left(50 \mu \mathrm{g} \mathrm{mL}^{-1}\right)$, and $5 \%$ sucrose. Following overnight growth, cultures were diluted 100 -fold into fresh DRM containing spectinomycin $\left(100 \mu \mathrm{g} \mathrm{mL}^{-1}\right)$ and carbenicillin $\left(50 \mu \mathrm{g} \mathrm{mL} L^{-1}\right)$. From the diluted cultures, $200 \mu \mathrm{L}$ of each culture were transferred to a 96-well black wall, clear bottom plate (Costar), topped with $20 \mu \mathrm{L}$ of mineral oil, and the $\mathrm{OD}_{600}$ was measured every $5 \mathrm{~min}$ over $15 \mathrm{~h}$. Separately, $400 \mu \mathrm{L}$ of each diluted culture were supplemented with kanamycin $\left(30 \mu \mathrm{g} \mathrm{mL}^{-1}\right)$ and grown in deepwell plates (VWR) at $37^{\circ} \mathrm{C}$ with shaking at $900 \mathrm{RPM}$. Colonies that survived selection in kanamycin were excluded from final analysis, as survival in kanamycin indicates persistence of the resident $\mathrm{pCSacB}$ plasmid (which carries a KanR resistance cassette). The doubling time of each culture was calculated using the Growthcurver package (version 0.3.0) ${ }^{61}$ in $R$ (version 3.5.2). Data for SQ171 assay complementation in the main text is reported as fitness in doublings/h, obtained by taking the inverse of doubling time in minutes and multiplying by 60 .

Phylogenetic analyses. To calculate sequence identities, $16 \mathrm{~S}$ sequences of all rRNAs used in the study were aligned using Clustal Omega with default settings ${ }^{48}$ The phylogenetic tree (Fig. 2d) was constructed using phylogenetic relationships derived from the Genome Taxonomy database (GTDB) ${ }^{62}$. In short, the entire bacterial GTDB phylogenetic tree (release 86.1) was downloaded from https://data ace.uq.edu.au/public/gtdb/data/releases/release86/86.1/. The phylogenetic tree was pruned to include only species of interest (see Supplementary Table 6 for the correspondence between species names and respective GTDB representative 
genomes) using the Ape package (version 5.3) in $R$ (version 3.5.2). The pairwise distances between the tips in the pruned trees were computed using the Ape package ${ }^{63}$. The tree was visualized using iTOL (version 5.7) ${ }^{64}$. Per-base conservation $^{41}$ (Fig. 3a) was calculated using the Biostrings (version 2.52.0) (https://bioconductor.org/packages/Biostrings) and TFBSTools ${ }^{65}$ (version 1.20.0) packages in $R$ (version 3.5.2).

Protein sequence similarity analysis. To analyze sequence similarities of r-proteins, RefSeq proteomes of relevant species were downloaded and a local BLAST database was created from these proteomes (using BLAST version 2.7.1). E. coli SSU r-protein sequences were queried against the database using local blastp with default parameters using the BLOSUM62 similarity matrix. Hits were filtered to those annotated with "30S," "SSU," or "ribosomal protein."

Reporting summary. Further information on research design is available in the Nature Research Reporting Summary linked to this article.

\section{Data availability}

The authors declare that all the data supporting the findings of this study are available within the paper and its supplementary information files. The source data for Figs. 1b-c, 2b-f, 3a, 3c-e, 4b-f, 5a-f, 1c-e, and Supplementary Figs. 2a-h, 3b-p, 4, 5a-e, 6a-e, 7a-c, 9a-j, 10a, 10c-f, and 11a-n are provided as a Source Data File. The Genome Taxonomy Data Base phylogenetic tree used for phylogenetic analysis can be downloaded at https://data. ace.uq.edu.au/public/gtdb/data/releases/release86/86.1/. See Supplementary Table 6 for the correspondence between species names, NCBI taxIDs, NCBI species taxIDs, NCBI strain identifiers, and respective GTDB representative genomes. Key plasmids used in this study have been deposited in Addgene (see Supplementary Table 8 for Addgene IDs). All other relevant data are available from the authors upon reasonable request. Source data are provided with this paper.

Received: 15 November 2020; Accepted: 16 December 2020; Published online: 26 January 2021

\section{References}

1. Chubukov, V., Gerosa, L., Kochanowski, K. \& Sauer, U. Coordination of microbial metabolism. Nat. Rev. Microbiol. 12, 327-340 (2014).

2. de Jong, H., Geiselmann, J. \& Ropers, D. Resource reallocation in bacteria by reengineering the gene expression machinery. Trends Microbiol. 25, 480-493 (2017).

3. Kaczanowska, M. \& Ryden-Aulin, M. Ribosome biogenesis and the translation process in Escherichia coli. Microbiol. Mol. Biol. Rev. 71, 477-494 (2007).

4. Rackham, O. \& Chin, J. W. A network of orthogonal ribosome x mRNA pairs. Nat. Chem. Biol. 1, 159-166 (2005).

5. Wang, K., Neumann, H., Peak-Chew, S. Y. \& Chin, J. W. Evolved orthogonal ribosomes enhance the efficiency of synthetic genetic code expansion. Nat. Biotechnol. 25, 770 (2007).

6. Neumann, H., Wang, K., Davis, L., Garcia-Alai, M. \& Chin, J. W. Encoding multiple unnatural amino acids via evolution of a quadruplet-decoding ribosome. Nature 464, 441 (2010).

7. Schmied, W. H. et al. Controlling orthogonal ribosome subunit interactions enables evolution of new function. Nature 564, 444-448 (2018).

8. Orelle, C. et al. Protein synthesis by ribosomes with tethered subunits. Nature 524, 119-124 (2015).

9. Fried, S. D., Schmied, W. H., Uttamapinant, C. \& Chin, J. W. Ribosome subunit stapling for orthogonal translation in E.coli. Angew. Chem. Int. Ed. Engl. 54, 12791-12794 (2015).

10. Filipovska, A. \& Rackham, O. Specialization from synthesis: how ribosome diversity can customize protein function. FEBS Lett. 587, 1189-1197 (2013).

11. Kurylo, C. M. et al. Endogenous rRNA sequence variation can regulate stress response gene expression and phenotype. Cell Rep. 25, 236-248.e6 (2018).

12. Song, W. et al. Divergent rRNAs as regulators of gene expression at the ribosome level. Nat. Microbiol. 4, 515-526 (2019).

13. Kim, H.-L. et al. Heterogeneous rRNAs are differentially expressed during the morphological development of Streptomyces coelicolor. FEMS Microbiol. Lett. 275, 146-152 (2019).

14. Moll, I., Resch, A. \& Blasi, U. Discrimination of 5'-terminal start codons by translation initiation factor 3 is mediated by ribosomal protein S1. FEBS Lett. 436, 213-217 (1998).

15. Deusser, E. \& Wittman, H.-G. Biological sciences: ribosomal proteins: variation of the protein composition in Escherichia coli ribosomes as function of growth rate. Nature 238, 269-270 (2019).

16. Kitahara, K. \& Miyazaki, K. Revisiting bacterial phylogeny: natural and experimental evidence for horizontal gene transfer of 16S rRNA. Mob. Genet. Elem. 3, e24210 (2013)
17. Kitahara, K., Yasutake, Y. \& Miyazaki, K. Mutational robustness of $16 \mathrm{~S}$ ribosomal RNA, shown by experimental horizontal gene transfer in Escherichia coli. Proc. Natl Acad. Sci. USA 109, 19220-19225 (2012).

18. Asai, T., Zaporojets, D., Squires, C. \& Squires, C. L. An Escherichia coli strain with all chromosomal rRNA operons inactivated: complete exchange of rRNA genes between bacteria. Proc. Natl Acad. Sci. USA 96, 1971-1976 (1999).

19. Gyorfy, Z. et al. Engineered ribosomal RNA operon copy-number variants of E. coli reveal the evolutionary trade-offs shaping rRNA operon number. Nucleic Acids Res. 43, 1783-1794 (2019).

20. Shi, X., Chiu, K., Ghosh, S. \& Joseph, S. Bases in 16S rRNA important for subunit association, tRNA binding, and translocation. Biochemistry 48, 6772-6782 (2009).

21. Gutgsell, N. S. \& Jain, C. Role of precursor sequences in the ordered maturation of E. coli 23S ribosomal RNA. RNA 18, 345-353 (2012).

22. Kitahara, K. \& Suzuki, T. The ordered transcription of RNA domains is not essential for ribosome biogenesis in Escherichia coli. Mol. Cell 34, 760-766 (2009).

23. Sahu, B., Khade, P. K. \& Joseph, S. Functional replacement of two highly conserved tetraloops in the bacterial ribosome. Biochemistry 51, 7618-7626 (2012).

24. Mylvaganam, S. \& Dennis, P. P. Sequence heterogeneity between the two genes encoding 16S rRNA from the halophilic archaebacterium Haloarcula marismortui. Genetics 130, 399-410 (1992).

25. Ueda, K., Seki, T., Kudo, T., Yoshida, T. \& Kataoka, M. Two distinct mechanisms cause heterogeneity of 16S rRNA. J. Bacteriol. 181, 78-82 (1999).

26. Yap, W. H., Zhang, Z. \& Wang, Y. Distinct types of rRNA operons exist in the genome of the actinomycete thermomonospora chromogena and evidence for horizontal transfer of an entire rRNA operon. J. Bacteriol. 181, 5201-5209 (1999).

27. Tian, R. M., Cai, L., Zhang, W. P., Cao, H. L. \& Qian, P. Y. Rare events of intragenus and intraspecies horizontal transfer of the 16S rRNA gene. Genome Biol. Evol. 7, 2310-2320 (2015).

28. Acinas, S. G., Marcelino, L. A., Klepac-Ceraj, V. \& Polz, M. F. Divergence and redundancy of $16 \mathrm{~S}$ rRNA sequences in genomes with multiple rrn operons. $J$. Bacteriol. 186, 2629-2635 (2004).

29. Prossliner, T., Skovbo Winther, K., Sorensen, M. A. \& Gerdes, K. Ribosome hibernation. Annu. Rev. Genet. 52, 321-348 (2018).

30. Byrgazov, K., Vesper, O. \& Moll, I. Ribosome heterogeneity: another level of complexity in bacterial translation regulation. Curr. Opin. Microbiol. 16, 133-139 (2013)

31. Hui, A. \& de Boer, H. A. Specialized ribosome system: preferential translation of a single mRNA species by a subpopulation of mutated ribosomes in Escherichia coli. Proc. Natl Acad. Sci. USA 84, 4762-4766 (1987).

32. Brink, M. F., Verbeet, M. P. \& de Boer, H. A. Specialized ribosomes: highly specific translation in vivo of a single targetted mRNA species. Gene 156, 215-222 (1995)

33. Hui, A., Jhurani, P. \& de Boer, H. A. Directing ribosomes to a single mRNA species: a method to study ribosomal RNA mutations and their effects on translation of a single messenger in Escherichia coli. Methods Enzymol. 153, 432-452 (1987).

34. Lee, K., Holland-Staley, C. A. \& Cunningham, P. R. Genetic analysis of the Shine-Dalgarno interaction: selection of alternative functional mRNA-rRNA combinations. RNA 2, 1270-1285 (1996).

35. Asai, T. et al. Construction and initial characterization of Escherichia coli strains with few or no intact chromosomal rRNA operons. J. Bacteriol. 181, 3803-3809 (1999).

36. Blattner, F. R. et al. The complete genome sequence of Escherichia coli K-12 Science 277, 1453-1462 (1997).

37. Traxler, M. F. et al. The global, ppGpp-mediated stringent response to amino acid starvation in Escherichia coli. Mol. Microbiol. 68, 1128-1148 (2008).

38. Mizoguchi, H., Tanaka-Masuda, K. \& Mori, H. A simple method for multiple modification of the Escherichia coli K-12 chromosome. Biosci. Biotechnol. Biochem. 71, 2905-2911 (2007).

39. Li, X. T., Thomason, L. C., Sawitzke, J. A., Costantino, N. \& Court, D. L. Positive and negative selection using the tet $\mathrm{A}-\mathrm{sacB}$ cassette: recombineering and P1 transduction in Escherichia coli. Nucleic Acids Res. 41, e204 (2013).

40. Pedelacq, J. D., Cabantous, S., Tran, T., Terwilliger, T. C. \& Waldo, G. S. Engineering and characterization of a superfolder green fluorescent protein. Nat. Biotechnol. 24, 79-88 (2006).

41. Valdar, W. S. Scoring residue conservation. Proteins 48, 227-241 (2002).

42. Deutscher, M. P. \& Biology, B. M. Chapter 9 maturation and degradation of ribosomal RNA in bacteria. Prog. Mol. Biol. Transl. Sci. 85, 369-391 (2019).

43. Shajani, Z., Sykes, M. T. \& Williamson, J. R. Assembly of bacterial ribosomes. Annu. Rev. Biochem. 80, 501-526 (2011).

44. Dunstan, M. S., Guhathakurta, D., Draper, D. E. \& Conn, G. L. Coevolution of protein and RNA structures within a highly conserved ribosomal domain. Chem. Biol. 12, 201-206 (2005).

45. Brandman, R., Brandman, Y. \& Pande, V. S. Sequence coevolution between RNA and protein characterized by mutual information between residue triplets. PLoS One 7, e30022 (2012). 
46. Peterson, J. \& Phillips, G. J. New pSC101-derivative cloning vectors with elevated copy numbers. Plasmid 59, 193-201 (2008).

47. Darlington, A. P. S., Kim, J., Jiménez, J. I. \& Bates, D. G. Dynamic allocation of orthogonal ribosomes facilitates uncoupling of co-expressed genes. Nat. Commun. 9, 1-12 (2018).

48. Madeira, F. et al. The EMBL-EBI search and sequence analysis tools APIs in 2019. Nucleic Acids Res. 47, W636-w641 (2019).

49. Noeske, J. et al. High-resolution structure of the Escherichia coli ribosome. Nat. Struct. Mol. Biol. 22, 336-341 (2015).

50. Cochella, L. \& Green, R. Isolation of antibiotic resistance mutations in the rRNA by using an in vitro selection system. Proc. Natl Acad. Sci. USA 101, 3786-3791 (2004).

51. Wang, H. H. \& Church, G. M. Multiplexed genome engineering and genotyping methods applications for synthetic biology and metabolic engineering. Methods Enzymol. 498, 409-426 (2011).

52. Datsenko, K. A. \& Wanner, B. L. One-step inactivation of chromosomal genes in Escherichia coli K-12 using PCR products. Proc. Natl Acad. Sci. USA 97, 6640-6645 (2000).

53. Gupta, P., Kannan, K., Mankin, A. S. \& Vazquez-Laslop, N. Regulation of gene expression by macrolide-induced ribosomal frameshifting. Mol. Cell 52, 629-642 (2013).

54. Shoji, S., Dambacher, C. M., Shajani, Z., Williamson, J. R. \& Schultz, P. G. Systematic chromosomal deletion of bacterial ribosomal protein genes. J. Mol. Biol. 413, 751-761 (2011).

55. Dabbs, E. R. Selection for Escherichia coli mutants with proteins missing from the ribosome. J. Bacteriol. 140, 734-737 (1979).

56. Held, W. A., Ballou, B., Mizushima, S. \& Nomura, M. Assembly mapping of $30 \mathrm{~S}$ ribosomal proteins from Escherichia coli. Further studies. J. Biol. Chem. 249, 3103-3111 (1974).

57. Sengupta, J., Agrawal, R. K. \& Frank, J. Visualization of protein S1 within the 30 S ribosomal subunit and its interaction with messenger RNA. Proc. Natl Acad. Sci. USA 98, 11991-11996 (2001).

58. Marzi, S. et al. Structured mRNAs regulate translation initiation by binding to the platform of the ribosome. Cell 130, 1019-1031 (2007).

59. Hubbard, B. P. et al. Continuous directed evolution of DNA-binding proteins to improve TALEN specificity. Nat. Methods 12, 939 (2015).

60. Carlson, J. C., Badran, A. H., Guggiana-Nilo, D. A. \& Liu, D. R. Negative selection and stringency modulation in phage-assisted constinuous evolution. Nat. Chem. Biol. 10, 216-222 (2014).

61. Sprouffske, K. \& Wagner, A. Growthcurver: an R package for obtaining interpretable metrics from microbial growth curves. BMC Bioinforma. 17, 172 (2016).

62. Parks, D. H. et al. A standardized bacterial taxonomy based on genome phylogeny substantially revises the tree of life. Nat. Biotechnol. 36, 996-1004 (2018).

63. Paradis, E. \& Schliep, K. ape 5.0: an environment for modern phylogenetics and evolutionary analyses in R. Bioinformatics 35, 526-528 (2019).

64. Letunic, I. \& Bork, P. Interactive Tree Of Life (iTOL) v4: recent updates and new developments. Nucleic Acids Res. 47, W256-w259 (2019).

65. Tan, G. \& Lenhard, B. TFBSTools: an R/bioconductor package for transcription factor binding site analysis. Bioinformatics 32, 1555-1556 (2016).

\section{Acknowledgements}

The authors would like to thank all members of the Badran lab for helpful discussions. This work was supported by the National Institutes of Health Director's Early Independence Award (DP5-OD-024590), the NASA Exobiology Program
(NNH17ZDA001N-EXO), and the Broad Institute of MIT and Harvard. The authors thank the following individuals for providing templates for rDNA amplification: Arnaud Guttierez, Bernardo Cervantez, Jim Gomez, Roby Bhattacharyya, Daria van Tyne, Austin Cole, Aaron James Dy, Allison Coe, Ning Mao, Nick Lyons, Becky Lamason, Kevin Gozzi, Vayu Maini Rekdal, Samantha Palace, Daniel Jun, and Jacob Cohen. The authors additionally thank the laboratories of Deborah Hung, Paul Blainey, and James Collins for equipment use and assistance. The authors are grateful to Erik Carlson and Mike Jewett for sharing materials used in this work.

\section{Author contributions}

N.S.K. designed the research, performed experiments, and analyzed data. R.F. designed, carried out, and analyzed SQ171 and sfGFP assays. S.B. performed rRNA and r-protein phylogenetic analyses. G.D.C. generated and analyzed fluorescent protein reporters. A.H B. conceived of and designed the research, performed experiments, analyzed data, and supervised the research. N.S.K. and A.H.B. wrote the manuscript, with contributions from all authors.

\section{Competing interests}

N.S.K. and A.H.B. declare competing interests. The Broad Institute has filed a patent application directed to work described in this article; N.S.K. and A.H.B. are inventors on that application. The provisional applications were filed in the United States, entitled "Heterologous Ribosome Generation, Assessment and Compositions Thereof". The associated PCT/US2020/041905, was filed on the 14th of July 2020. This patent covers methods for generating heterologous ribosomes and assessing/improving their activity. R.F., S.B., and G.D.C. declare no competing interests.

\section{Additional information}

Supplementary information is available for this paper at https://doi.org/10.1038/s41467 020-20759-z.

Correspondence and requests for materials should be addressed to A.H.B

Peer review information Nature Communications thanks the anonymous reviewer(s) for their contribution to the peer review of this work. Peer reviewer reports are available.

Reprints and permission information is available at http://www.nature.com/reprints

Publisher's note Springer Nature remains neutral with regard to jurisdictional claims in published maps and institutional affiliations.

Open Access This article is licensed under a Creative Commons Attribution 4.0 International License, which permits use, sharing, adaptation, distribution and reproduction in any medium or format, as long as you give appropriate credit to the original author(s) and the source, provide a link to the Creative Commons license, and indicate if changes were made. The images or other third party material in this article are included in the article's Creative Commons license, unless indicated otherwise in a credit line to the material. If material is not included in the article's Creative Commons license and your intended use is not permitted by statutory regulation or exceeds the permitted use, you will need to obtain permission directly from the copyright holder. To view a copy of this license, visit http://creativecommons.org/ licenses/by/4.0/

(C) The Author(s) 2021 